\title{
Coupling impedance and wake functions for laminated structures with an application to the Fermilab Booster
}

\author{
Alexandru Macridin, Panagiotis Spentzouris, and James Amundson \\ Fermilab, P.O. Box 500, Batavia, Illinois 60510, USA \\ Linda Spentzouris and Daniel McCarron \\ Illinois Institute of Technology, Chicago, Illinois 60616, USA \\ (Received 8 April 2011; published 28 June 2011)
}

\begin{abstract}
We calculate the impedance and wake functions for laminated structures with parallel-plane and circular geometries in the ultrarelativistic limit. We critically examine the approximations used in the literature for the coupling impedance in laminated chambers and find that most of them are not justified because the wall surface impedance is large. A comparison between flat and circular geometry impedances is presented. We apply our calculation in a state-of-the-art beam dynamics simulation of the Fermilab Booster which includes nonlinear optics, laminated wakefields, and space charge impedance. The latter can have a significant effect away from the ultrarelativistic limit. Even though the simulations and the comparison with the experiment are done at the Booster injection energy, where the relativistic factor $\gamma=1.42$, we find good agreement between our calculation of the coherent tune shift and recent experimental measurements.
\end{abstract}

DOI: 10.1103/PhysRevSTAB.14.061003

PACS numbers: 29.20.-c, 29.27.Bd

\section{INTRODUCTION}

Wakefields are an important component of beam dynamics in high-intensity accelerators, being a potential cause for losses and instabilities. While there is satisfactory understanding of wakefields in metallic chambers with smooth geometries, the attempts to describe the impedance effect in structures with laminations are not in very good agreement with experimental measurements. Magnetic elements for guiding and focusing beams are often constructed from stacks of iron laminations alternating with the layers of epoxy holding them together. In the absence of beam pipe the presence of laminations produces large wake effects. Because of the strong demand for increasing intensity in machines with laminated chambers, such as the Fermilab Booster synchrotron, theoretical investigation of wake effects in the presence of laminations is of paramount importance.

In addition to improved calculations of the effects of wakefields, realistic simulations which consider the interplay between wakefields and other effects in accelerators must be employed to accurately describe beam dynamics and to compare with measurements. While most of the analytic investigations of wake effects have been done in the frequency domain and require an impedance function over some finite frequency range, beam simulation algorithms require point-charge wake functions in the

Published by the American Physical Society under the terms of the Creative Commons Attribution 3.0 License. Further distribution of this work must maintain attribution to the author(s) and the published article's title, journal citation, and DOI. time-and-space domain. The impedance calculation is in general much easier; the wakefields are related to the impedance via a Fourier transform. However, this method requires knowledge of the impedance over a large frequency domain as well as the asymptotic behavior at both small and large frequencies.

In this paper we calculate the impedance and the wake functions for laminated structures with parallel-plane and circular geometries for ultrarelativistic beams. First, the coupling impedance is derived as a function of the wall surface impedance. Then the surface impedance is calculated by solving the Maxwell equations inside the lamination and the crack regions. A critical analysis of the resistive-wall impedance for metallic chambers and the approximations involved in its derivations is also presented.

The Fermilab Booster is a good example of a machine where the beam is exposed to laminations. It is currently running with beam intensities roughly twice its design value. Direct measurements using a stretched wire between the gradient magnets ends indicate a large longitudinal coupling impedance [1]. The coherent tune shift, which shows horizontal tune increase and vertical tune decrease, presents strong evidence for large transverse wakes [2]. Since the effect on the transverse tunes is specific to geometries without circular symmetries [3], these measurements also stress the importance of choosing the right chamber geometry for any calculation which addresses the wakes in the Booster magnets.

There are several calculations of the impedance due to laminated structures with applications for the Fermilab Booster [4-7]. Most of them [4-6] address only circular geometry, which is a poor approximation for the Booster 
magnets. Moreover, the resistive-wall specific relation they use to connect the wall surface impedance to the coupling impedance is not valid for structures with large surface impedance and flat chambers such as the laminated magnets in the Booster. Even for the circular geometry they address only the longitudinal impedance for uniform round beams, i.e., only the $m=0$ angular channel. However, since the transverse Lorentz force vanishes identically for the lowest rotational symmetric mode $m=0$, calculations in the $m=1$ channel are necessary to address the transverse impedance $[8,9]$. Note that the commonly used relation for a rough estimation of the transverse impedance as a function of the longitudinal one, based on dimensionality arguments [8], is not valid for the laminated structures. $\mathrm{Ng}$ [7] addresses both the parallel-plane geometry, which is close to the real geometry of the Booster magnets [10], and the transverse impedance. However, for the sake of simplicity, he assumes a beam which extends to infinity in the horizontal direction. While this approximation works for metallic pipes, it produces wakes which are too large for laminated chambers. In parallel with our work, an independent derivation of the impedance in laminated structures was done by Burov and Lebedev in [11]. Their results, albeit derived in a different way, are in agreement with ours.

We employ the SYNERGIA code to run realistic beam simulations which include wake interactions specific to laminated structures. SYNERGIA is an extensible beam dynamics framework developed at Fermilab $[12,13]$ which incorporates a large collection of physical models, specialized modules, and numerical libraries. SYNERGIA simulates complex beam dynamics, employing high order maps to describe single-particle propagation through accelerators and considers collective effects such as space charge forces and wakefield interactions. Our SYNERGIA simulations of the Booster with wakefields predict transverse tune shifts in good agreement with experiment [2].

The paper is organized as follows. In Sec. II we calculate the impedance and the wake functions for flat chambers with laminations. We discuss the approximations involved in the calculation at length. In Sec. III we perform a similar calculation for circular chambers with laminations and compare the results between the round and the flat chambers. We then employ SYNERGIA for simulations of the beam dynamics in the Fermilab Booster and compare the results with experimental data in Sec. IV.

\section{PARALLEL-PLANE CHAMBER WITH LAMINATIONS}

\section{A. Wake functions}

The wake functions describe the effect of the electromagnetic field created by a particle moving through an accelerator beam pipe upon the trailing particles. Consider an ultrarelativistic beam flowing along the $z$ direction between two parallel plates horizontally aligned. If the distance between the leading and trailing particle is $|z|$, the momentum of the trailing particle traversing a structure of length $L$ will be modified by $[3,14]$

$$
\begin{gathered}
c \Delta p_{z}=-q Q W^{\|}(z) \\
c \Delta p_{x}=-q Q\left[W_{x}^{\perp}(z) X-W_{x}^{\perp}(z) x\right] \\
c \Delta p_{y}=-q Q\left[W_{y}^{\perp}(z) Y+W_{x}^{\perp}(z) y\right] .
\end{gathered}
$$

Here $Q(q)$ and $(X, Y)[(x, y)]$ represent the charge and the transverse displacement of the leading (trailing) particle, respectively. $\|$ and $\perp$ denote the longitudinal and the transverse directions. The higher-order terms in the displacement are neglected. For this particular geometry, only two wake functions, $W_{x}^{\perp}(z)$ and $W_{y}^{\perp}(z)$, are needed for the transverse directions [Eqs. (2) and (3)]. This is a consequence of the translational symmetry along the horizontal direction and of the Panofsky-Wenzel theorem which requires that $\frac{\partial \Delta p_{x}}{\partial x}=-\frac{\partial \Delta p_{y}}{\partial y}$. The terms proportional to the displacement of the leading particle, i.e., to $X$ or $Y$, are called dipole wakes while the ones proportional to the displacement of the trailing particle, i.e., to $x$ or $y$, are called quadrupole wakes [15]. Note that in a circular pipe and ultrarelativistic limit the quadrupole wakefields vanish due to symmetry, while extra terms can be present in pipes with lower symmetries [14].

To calculate the electromagnetic field, often it is easier to solve the Maxwell equations in the frequency domain, and afterward calculate the impedances. Once the impedances are known, the wakes can be obtained by a Fourier transform,

$$
\begin{gathered}
W^{\|}(z)=\frac{1}{2 \pi} \int_{-\infty}^{\infty} d \omega Z^{\|}(\omega) e^{-j(\omega / c) z} \\
W_{(x, y)}^{\perp}(z)=\frac{j}{2 \pi} \int_{-\infty}^{\infty} d \omega Z_{(x, y)}^{\perp}(\omega) e^{-j(\omega / c) z} .
\end{gathered}
$$

In order to calculate the Fourier transforms, it is necessary to have accurate knowledge of the impedance $Z(\omega)$ over the whole frequency domain.

\section{B. Impedance}

The ultrarelativistic coupling impedance is given by the solution of the Maxwell equations for an ultrarelativistic beam in a pipe with finite conductivity. The purpose of this section is to write the coupling impedance as a function of the wall surface impedances for chamber with flat geometry. The surface impedances are given by the ratio of the different tangential components of the electric and the magnetic fields at the chamber's walls and are specific to the chamber. In the next two sections we will derive the surface impedances for the metallic and laminated chambers, respectively. 
Our derivation follows closely $\mathrm{Ng}$ 's derivation [7]. Consider a ultrarelativistic beam propagating through the pipe with a vertical offset $y=a$. The distance between the parallel plates is $2 b$. The charge density and the electrical current are given by

$$
\begin{gathered}
\rho(x, y, z, t)=\rho \delta(x) \delta(y-a) e^{j(\omega t-k z)} \\
\vec{J}(x, y, z, t)=\rho(x, y, z, t) c \hat{z}
\end{gathered}
$$

with $k=\frac{\omega}{c}$. We look for synchronous solutions of the Maxwell equations:

$$
\begin{aligned}
\vec{E}(x, y, z, t) & =\vec{E}(x, y) e^{j(\omega t-k z)} \\
\vec{H}(x, y, z, t) & =\vec{H}(x, y) e^{j(\omega t-k z)} .
\end{aligned}
$$

The spectral decomposition along the horizontal direction can be written as

$$
\begin{aligned}
& \quad\left[E_{z}(x, y), E_{y}(x, y), H_{x}(x, y)\right] \\
& \quad=\int_{-\infty}^{\infty}\left[E_{z}(\eta, y), E_{y}(\eta, y), H_{x}(\eta, y)\right] \cos (\eta x) d \eta \\
& {\left[H_{z}(x, y), H_{y}(x, y), E_{x}(x, y)\right]} \\
& \quad=\int_{-\infty}^{\infty}\left[H_{z}(\eta, y), H_{y}(\eta, y), E_{x}(\eta, y)\right] \sin (\eta x) d \eta .
\end{aligned}
$$

Inside the vacuum chamber, from the transverse components of the Maxwell equations, $\nabla \times E=-j k Z_{0} H$ and $\nabla \times H=j k E$, one gets

$$
\begin{aligned}
& \partial_{y} E_{z}=\eta Z_{0} H_{z} \\
& \partial_{y} Z_{0} H_{z}=\eta E_{z},
\end{aligned}
$$

which results in

$$
\begin{gathered}
E_{z}(\eta, y)=A_{\eta} \cosh \eta y+\bar{A}_{\eta} \sinh \eta y \\
Z_{0} H_{z}(\eta, y)=A_{\eta} \sinh \eta y+\bar{A}_{\eta} \cosh \eta y,
\end{gathered}
$$

where $Z_{0} \approx 377 \Omega$ is the free space impedance. The transverse components of the fields can also be easily deduced. For a detailed derivation see $\mathrm{Ng}$ [7]. The set of constants $A_{\eta}$ and $\bar{A}_{\eta}$ are determined by imposing boundary conditions on the field components at the pipe's walls. They are also uniquely determined if the surface impedances

$$
\begin{aligned}
& \mathcal{R}_{z}(\eta)= \pm\left.\frac{E_{z}(\eta)}{H_{x}(\eta)}\right|_{y= \pm b} \\
& \mathcal{R}_{x}(\eta)=\left.\mp \frac{E_{x}(\eta)}{H_{z}(\eta)}\right|_{y= \pm b}
\end{aligned}
$$

at the pipe's walls are known, by substituting in the expressions for the fields and solving for the coefficients,
$A_{\eta}=\frac{-\frac{\rho \mathcal{R}_{z}(\eta)}{2 Z_{0} \epsilon_{0}} \operatorname{sech}^{2} \eta b \cosh \eta a}{1+j \frac{\mathcal{R}_{z}(\eta)}{Z_{0}}\left(\frac{k}{\eta}-\frac{\eta}{k}\right) \tanh \eta b+\frac{\mathcal{R}_{z}(\eta) \mathcal{R}_{x}(\eta)}{Z_{0}^{2}} \tanh ^{2} \eta b}$

$\bar{A}_{\eta}=\frac{-\frac{\rho \mathcal{R}_{z}(\eta)}{2 Z_{0} \epsilon_{0}} \operatorname{csch}^{2} \eta b \sinh \eta a}{1+j \frac{\mathcal{R}_{z}(\eta)}{Z_{0}}\left(\frac{k}{\eta}-\frac{\eta}{k}\right) \operatorname{coth} \eta b+\frac{\mathcal{R}_{z}(\eta) \mathcal{R}_{x}(\eta)}{Z_{0}^{2}} \operatorname{coth}^{2} \eta b}$.

Now the problem of determining the constants $A_{\eta}$ and $\bar{A}_{\eta}$ has changed into that of determining $\mathcal{R}_{z}$ and $\mathcal{R}_{x}$ from the boundary conditions.

The longitudinal and the transverse impedances can be written as a function of the longitudinal fields $E_{z}$ and $H_{z}$. Thus, the longitudinal impedance is

$$
\begin{aligned}
Z^{\|}= & -\frac{\left.E_{z}(x=0, y=0)\right|_{a=0}}{\rho c}=-\frac{1}{2 \pi \rho c} \int_{-\infty}^{\infty} d \eta A_{\eta} \\
= & \frac{1}{2 \pi} \\
& \times \int_{0}^{\infty} d \eta \frac{\operatorname{sech}^{2} \eta b \mathcal{R}_{z}(\eta)}{1+j \frac{\mathcal{R}_{z}(\eta)}{Z_{0}}\left(\frac{k}{\eta}-\frac{\eta}{k}\right) \tanh \eta b+\frac{\mathcal{R}_{z}(\eta) \mathcal{R}_{x}(\eta)}{Z_{0}^{2}} \tanh ^{2} \eta b},
\end{aligned}
$$

provided the surface impedances are even functions of $\eta$.

The vertical force acting upon a trailing charge $q$ at $y=0$ created by a beam with displacement $y=a$ is

$$
\begin{aligned}
F_{y}(\eta, y=0) & =q\left[E_{y}(\eta)+Z_{0} H_{x}(\eta)\right]=q j \frac{\eta}{k} Z_{0} H_{z}(\eta) \\
& =q j \frac{\eta}{k} \bar{A}_{\eta} .
\end{aligned}
$$

The vertical impedance is

$$
\begin{aligned}
Z_{y}= & -\frac{F_{y}(x=0, y=0)}{j q \rho c a} \\
= & \frac{1}{2 \pi k} \\
& \times \int_{0}^{\infty} d \eta \frac{\eta^{2} \operatorname{csch}^{2} \eta b \mathcal{R}_{z}(\eta)}{1+j \frac{\mathcal{R}_{z}(\eta)}{Z_{0}}\left(\frac{k}{\eta}-\frac{\eta}{k}\right) \operatorname{coth} \eta b+\frac{\mathcal{R}_{z}(\eta) \mathcal{R}_{x}(\eta)}{Z_{0}^{2}} \operatorname{coth}^{2} \eta b},
\end{aligned}
$$

where only the first order term in the beam displacement was retained, i.e. $\sinh \eta a \approx \eta a$.

The horizontal force acting upon a trailing charge $q$ at $y=0$ by a centered beam is

$$
\begin{aligned}
F_{x}(\eta, y=0) & =q\left[E_{x}(\eta)-Z_{0} H_{y}(\eta)\right]=q j \frac{\eta}{k} E_{z}(\eta) \\
& =\left.q j \frac{\eta}{k} A_{\eta}\right|_{a=0} .
\end{aligned}
$$

For small horizontal displacement of the trailing particle, $F_{x}(x=\Delta) \approx \Delta \frac{\partial F_{x}}{\partial x}(x=0)$. Note that $F_{x}(x) \propto \sin \eta x$ thus, $\frac{\partial F_{x}}{\partial x} \propto \eta \cos \eta x$. The horizontal impedance is 


$$
\begin{aligned}
Z_{x}= & -\frac{\Delta \frac{\partial F_{x}}{\partial x}(x=0, y=0)}{j q \rho c \Delta} \\
= & \frac{1}{2 \pi k} \\
& \times \int_{0}^{\infty} d \eta \frac{\eta^{2} \operatorname{sech}^{2} \eta b \mathcal{R}_{z}(\eta)}{1+j \frac{\mathcal{R}_{z}(\eta)}{Z_{0}}\left(\frac{k}{\eta}-\frac{\eta}{k}\right) \tanh \eta b+\frac{\mathcal{R}_{z}(\eta) \mathcal{R}_{x}(\eta)}{Z_{0}^{2}} \tanh ^{2} \eta b} .
\end{aligned}
$$

The integrands in Eqs. (19), (21), and (23) are proportional to $\operatorname{sech}^{2} \eta b$ or $\operatorname{csch}^{2} \eta b$, thus going exponentially to zero for $\eta>\frac{1}{b}$. Therefore only the regime of small $\eta$, where $\eta<\frac{1}{b}$, is relevant for the impedance.

\section{Resistive-wall impedance}

The resistive-wall impedance for metallic flat pipes has been calculated by various authors $[7,16]$. The main reason for presenting the derivation here is to take a critical look at the approximations involved. As we will see in Sec. II D, many of the assumptions valid for the metallic pipes do not hold when laminations are present.

The homogeneous Maxwell equations imply

$$
\left(\frac{\partial^{2}}{\partial y^{2}}-k^{2}-\eta^{2}+\omega^{2} \epsilon \mu\right)\left(\begin{array}{c}
E_{z}(\eta, y, k, \omega) \\
H_{z}(\eta, y, k, \omega)
\end{array}\right)=0 .
$$

The solution for the fields inside the metallic pipe can be obtained by replacing the electric permittivity with

$$
\epsilon \rightarrow \epsilon_{0}\left(\epsilon_{r}+\frac{\sigma}{j \omega \epsilon_{0}}\right)
$$

where $\sigma$ is the pipe conductivity and $\epsilon_{r}$ is the relative permittivity. One gets

$$
\frac{\partial^{2}}{\partial y^{2}}\left(\begin{array}{c}
E_{z} \\
H_{z}
\end{array}\right)=\left(\eta^{2}+\lambda_{c}^{2}+p^{2}\right)\left(\begin{array}{c}
E_{z} \\
H_{z}
\end{array}\right)
$$

where $\lambda_{c}^{2}=j \omega \sigma \mu=j k Z_{0} \sigma \mu_{r}, p^{2}=k^{2}\left(1-\epsilon_{r} \mu_{r}\right)$, and $\mu_{r}$ is the relative permeability. The solution for an infinitely thick pipe is [7]

$$
\begin{aligned}
E_{z}= & A_{\eta} \cosh \eta b e^{\lambda(b \mp y)} \quad Z_{0} H_{z}= \pm A_{\eta} \sinh \eta b e^{\lambda(b \mp y)} \\
E_{x}= & \frac{A_{\eta}}{Z_{0} \sigma \mu_{r}-j k\left(1-\epsilon_{r} \mu_{r}\right)}\left(-\eta \cosh \eta b-\mu_{r} \lambda \sinh \eta b\right) \\
& \times e^{\lambda(b \mp y)} \\
Z_{0} H_{x}= & \frac{A_{\eta}}{Z_{0} \sigma \mu_{r}-j k\left(1-\epsilon_{r} \mu_{r}\right)}( \pm \lambda \cosh \eta b \pm \eta \sinh \eta b) \\
& \times e^{\lambda(b \mp y)} \mp \frac{j \lambda A_{\eta}}{k} \frac{Z_{0} \sigma}{Z_{0} \sigma \mu_{r}-j k\left(1-\epsilon_{r} \mu_{r}\right)} \\
& \times \cosh \eta b e^{\lambda(b \mp y)}
\end{aligned}
$$

where $\lambda=\sqrt{\eta^{2}+\lambda_{c}^{2}+p^{2}}$. For surface impedance, one gets

$$
\begin{aligned}
\frac{Z_{0}}{\mathcal{R}_{z}(\eta)}= & \frac{1}{Z_{0} \sigma \mu_{r}-j k\left(1-\epsilon_{r} \mu_{r}\right)}(\lambda+\eta \tanh \eta b) \\
& -\frac{j \lambda}{k} \frac{Z_{0} \sigma}{Z_{0} \sigma \mu_{r}-j k\left(1-\epsilon_{r} \mu_{r}\right)} \\
\frac{\mathcal{R}_{x}(\eta)}{Z_{0}}= & \frac{1}{Z_{0} \sigma \mu_{r}-j k\left(1-\epsilon_{r} \mu_{r}\right)}\left(\eta \operatorname{coth} \eta b+\mu_{r} \lambda\right) .
\end{aligned}
$$

The coupling impedances follow immediately by putting Eqs. (28) and (29) into Eqs. (19), (21), and (23).

Because of the large value of the conductivity in metallic pipes, $Z_{0} \sigma \approx 10^{8}-10^{9} \mathrm{~m}^{-1}$, several simplifying approximations are commonly used. $Z_{0} \sigma \mu_{r} \gg k\left(1-\epsilon_{r} \mu_{r}\right)$ up to very high frequencies, $\omega<\approx 10^{6} \mathrm{GHz}$. Thus, the term $j k\left(1-\epsilon_{r} \mu_{r}\right)$ can be neglected. As pointed out at the end of Sec. II B, only values of $\eta<\frac{1}{b} \approx 10^{2} \mathrm{~m}^{-1}$ are relevant, provided the pipe radius is of the order of centimeters. Thus,

$$
\lambda \approx \lambda_{c}=\frac{1+j}{\delta}
$$

where $\delta=\sqrt{\frac{2}{\omega \sigma \mu}}$ is the skin penetration depth. The first two terms in Eq. (28) are $\mathcal{O}\left(\frac{1}{Z_{0} \sigma}\right)$ relative to the third one, and thus can be neglected. In addition, the first term in Eq. (29) is $\mathcal{O}\left(\frac{1}{\sqrt{Z_{0} \sigma}}\right)$ relative to the second one. Therefore

$$
\mathcal{R}_{z}(\eta), \quad \mathcal{R}_{x}(\eta) \approx \frac{\lambda_{c}}{\sigma}=\frac{1+j}{\delta \sigma} .
$$

The surface impedances are small, $\mathcal{O}\left(\frac{1}{\sqrt{\sigma}}\right)$. For this reason the higher-order terms in the surface impedances in the denominator of Eqs. (19), (21), and (23) are not very important in the relevant frequency region for the beam dynamics. The term proportional to $\mathcal{R}_{z}(\eta) \mathcal{R}_{x}(\eta)$ is negligible. The term proportional to $\frac{\mathcal{R}_{z}}{Z_{0}} \frac{k}{\eta}$ determines the high frequency behavior $\left(k>\approx \frac{Z_{0}}{b \mathcal{R}_{z}}\right)$ and the one proportional to $\frac{\mathcal{R}_{z}}{Z_{0}} \frac{\eta}{k}$ the low frequency behavior $\left(k<\approx \frac{\mathcal{R}_{z}}{b Z_{0}}\right)$. These terms proportional to $\mathcal{R}_{z}$ are also small and are often neglected. Thus,

$$
\begin{gathered}
Z^{\|}=\frac{1}{2 \pi} \int_{0}^{\infty} d \eta \operatorname{sech}^{2} \eta b \mathcal{R}_{z}(\eta), \\
Z_{x}=\frac{1}{2 \pi k} \int_{0}^{\infty} d \eta \eta^{2} \operatorname{sech}^{2} \eta b \mathcal{R}_{z}(\eta), \\
Z_{y}=\frac{1}{2 \pi k} \int_{0}^{\infty} d \eta \eta^{2} \operatorname{csch}^{2} \eta b \mathcal{R}_{z}(\eta) .
\end{gathered}
$$

Since $\mathcal{R}_{z}$ is weakly dependent on $\eta$, the common approximations used in the literature for studying impedance effects in metallic pipes are

$$
Z^{\|}=\frac{\mathcal{R}_{z}}{2 \pi b},
$$




$$
\begin{aligned}
Z_{x} & =\frac{\mathcal{R}_{z}}{2 \pi k} \frac{\pi^{2}}{12 b^{3}}, \\
Z_{y} & =\frac{\mathcal{R}_{z}}{2 \pi k} \frac{\pi^{2}}{6 b^{3}},
\end{aligned}
$$

with $\mathcal{R}_{z}(\eta) \approx \mathcal{R}_{z}(\eta=0)$. In this approximation $Z^{\|} \propto$ $\sqrt{\omega}$, while the $Z_{x, y} \propto \frac{1}{\sqrt{\omega}}$ and diverges when $\omega \rightarrow 0$. If the low frequency behavior is important, one can employ

$$
\begin{aligned}
& Z_{x}=\frac{\mathcal{R}_{z}}{2 \pi k} \int_{0}^{\infty} d \eta \frac{\eta^{2} \operatorname{sech}^{2} \eta b}{1-\frac{j \mathcal{R}_{z} \eta}{Z_{0} k} \tanh \eta b}, \\
& Z_{y}=\frac{\mathcal{R}_{z}}{2 \pi k} \int_{0}^{\infty} d \eta \frac{\eta^{2} \operatorname{csch}^{2} \eta b}{1-\frac{j \mathcal{R}_{z} \eta}{Z_{0} k} \operatorname{coth} \eta b},
\end{aligned}
$$

which removes the low frequency divergence and makes the real part of the transverse impedance to go to zero and the imaginary part to approach a constant when $\omega \rightarrow 0$.

The impedance of an iron pipe with $b=2 \mathrm{~cm}$ and the commonly employed approximations for a metallic pipe discussed previously are illustrated in Fig. 1. Figures 1(a) and 1(b) show the longitudinal impedance and the approximations employed by Eq. (35) which holds up to $10 \mathrm{GHz}$. The horizontal and the vertical impedances and the approximations given by Eqs. (36) and (37) are shown in Figs. 1(c) and 1(d). The approximations are valid in the frequency interval $1 \mathrm{MHz}-2 \mathrm{GHz}$ which corresponds to $8 \mathrm{~mm}-16 \mathrm{~m}$ in the reciprocal space. One concludes that the commonly used approximations for resistive-wall

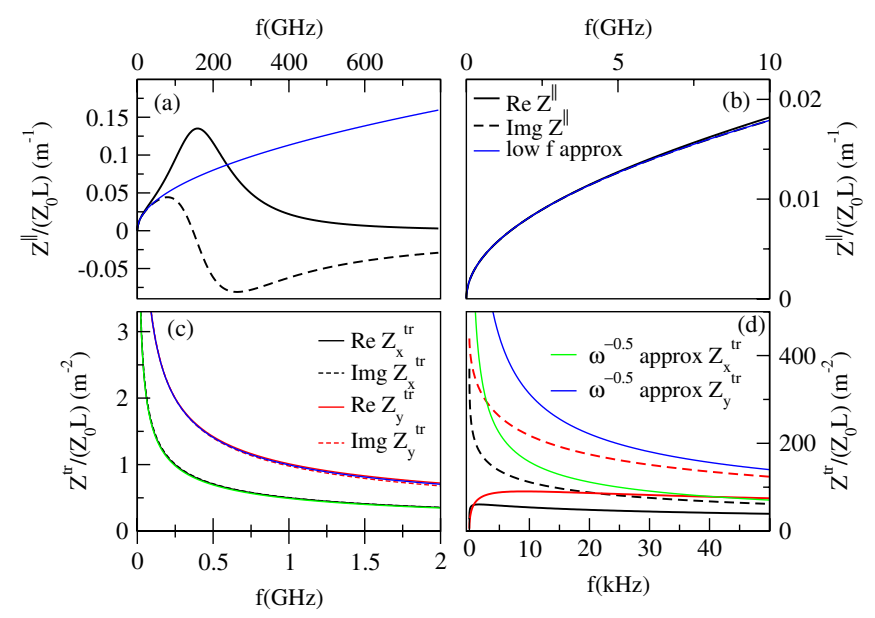

FIG. 1. Impedances of a parallel-plane iron pipe, $\sigma_{\text {iron }}=$ $0.5 \times 10^{7}(\Omega \mathrm{m})^{-1}$ with $b=0.02 \mathrm{~m}$. (a) and (b) Real (black continuous) and imaginary (black dashed) longitudinal impedance, Eq. (19). The low frequency approximation (blue) $\propto \sqrt{\omega}$, Eq. (35), is valid up to $10 \mathrm{GHz}$. (c) and (d) Real and imaginary part of the horizontal (black continuous and black dashed) and the vertical (red continuous and red dashed) impedances, Eqs. (23) and (21). Equation (36) (green) and Eq. (37) (blue) are valid approximations in the interval range $1 \mathrm{MHz}-2 \mathrm{GHz}$.

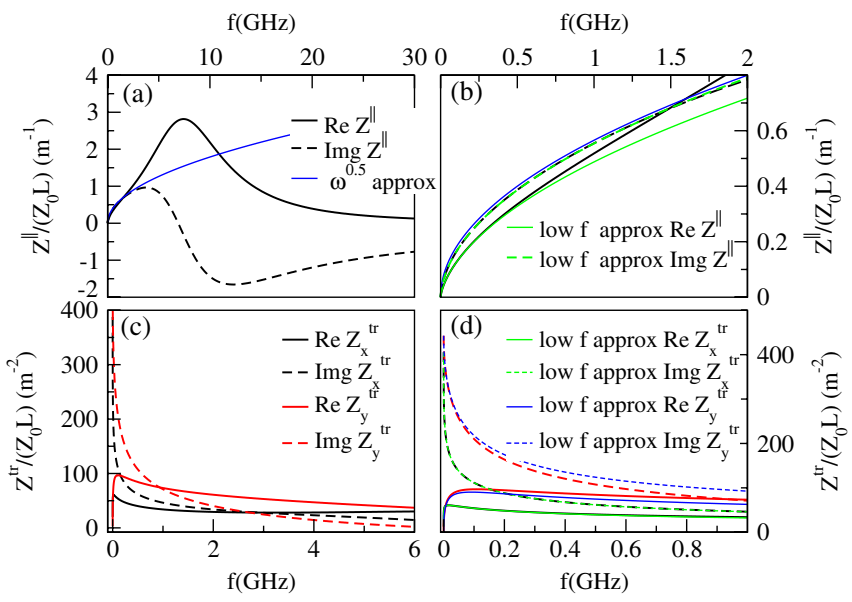

FIG. 2. Impedances of a parallel-plane pipe with $\sigma=$ $\sigma_{\text {iron }} \times 10^{-4}$ with $b=0.02 \mathrm{~m}$. (a) and (b) Real (black continuous) and imaginary (black dashed) longitudinal impedance, Eq. (19). The low frequency approximation (blue) $\propto \sqrt{\omega}$, Eq. (35) (blue) is not valid, while Eq. (40) (green) approximation hold up to $500 \mathrm{MHz}$. (c) and (d) Real and imaginary part of the horizontal (black continuous and black dashed) and the vertical (red continuous and red dashed) impedances, Eqs. (23) and (21). Equations (36) and (37) are not valid approximations, while Eq. (38) (green) and Eq. (39) (blue) are good only up to $200 \mathrm{MHz}$.

impedance are valid to investigate the wake effects in metallic pipes at distances of the order of centimeters and meters.

However, when the surface impedance is large the resistive-wall metallic pipe approximations, i.e., Eqs. (35)(37), are not valid anymore. Since in general the laminations increase the surface impedance by more than 2 orders of magnitude, this is an important aspect which should be accounted for when calculating the impedance in laminated structures. In Fig. 2 the impedance is plotted for a pipe with a much smaller conductivity than iron, $\sigma=\sigma_{\text {iron }} \times 10^{-4}$. According to Eq. (31) this corresponds to a 100 times larger surface impedance. The approximation $Z^{\|} \propto \sqrt{\omega}$ [Eq. (35)] commonly used for the longitudinal impedance, does not hold. A better low frequency approximation,

$$
Z^{\|}=\frac{1}{2 \pi} \int_{0}^{\infty} d \eta \frac{\operatorname{sech}^{2} \eta b \mathcal{R}_{z}(\eta)}{1-j \frac{\mathcal{R}_{z}(\eta)}{Z_{0}} \frac{\eta}{k} \tanh \eta b},
$$

can be used, but only up to $\simeq 500 \mathrm{MHz}$. Equations (36) and (37) do not work for the transverse impedance either. One can see in Fig. 2(c) that the real and the imaginary part of the transverse impedances are different. Equations (38) and (39) hold only below $200 \mathrm{MHz}$. The only term which can be safely neglected in the denominator of Eqs. (19), (21), and (23) is the one proportional to $\mathcal{R}_{z} \mathcal{R}_{x}$.

\section{Laminated structures}

A chamber with parallel faces and a laminated structure is sketched in Fig. 3. The subscript "1" denotes the 


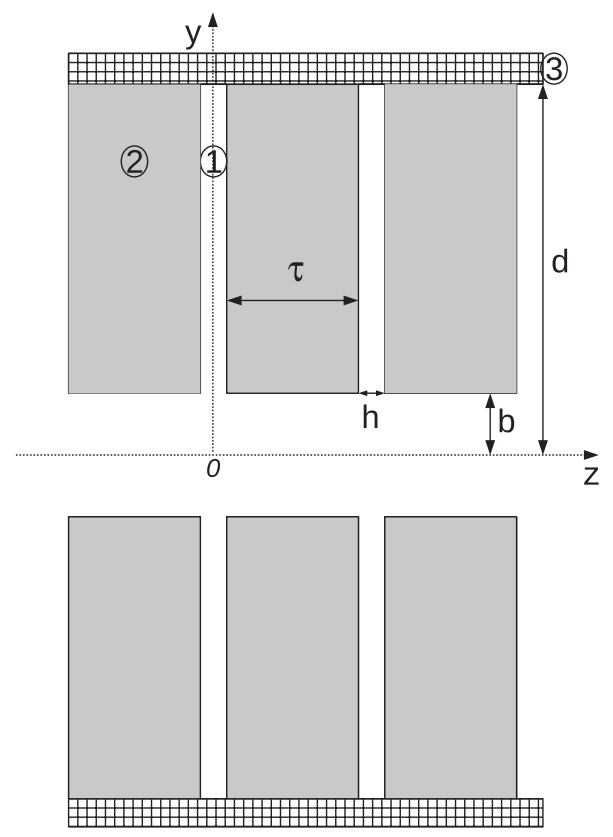

FIG. 3. Parallel-plane beam chamber with laminations. Subscript " 1 " denotes the crack and subscript " 2 " the lamination. The laminations are shorted by an ideal conductor " 3 ." The beam flows in the $z$ direction; the $x$ direction is perpendicular to the plane of the page.

dielectric crack of width $h$, and " 2 " denotes the metallic lamination of width $\tau$. The conductivity is large in the laminations $\sigma_{2}\left[\approx 10^{7}(\Omega \mathrm{m})^{-1}\right]$; it is assumed that the dimension $\tau+h$ is small $\left(\approx 10^{-3}-10^{-4} \mathrm{~m}\right)$ compared to the propagation wavelength.

With the above assumptions, the formalism presented in Sec. II B is valid and the coupling impedance can be written as a function of the chamber surface impedance. To show this, we start with the following definition of the longitudinal impedance [17]:

$$
Z^{\|}(\omega)=-\left.\frac{1}{\rho c} \int d z E_{z}(z, \omega)\right|_{x, y=0} e^{-j(\omega / c) z}
$$

Since the longitudinal dimension of laminations and cracks is small compared to the propagation wavelength, i.e. $\frac{\omega}{c}(\tau+h) \ll 1$, the following approximation is valid

$Z^{\|}(\omega) \approx-\left.\frac{1}{\rho c} \sum_{n=-\infty}^{\infty}(\tau+h) \bar{E}_{z}\left(z_{n}, \omega\right)\right|_{x, y=0} e^{-j(\omega / c) z_{n}}$,

where $z_{n}=n(\tau+h)$ is chosen to be in the middle of the crack " $n$," and

$$
\begin{aligned}
\bar{E}_{z}\left(z_{n}, \omega\right)= & \frac{1}{\tau+h}\left(\int_{z_{n}-h / 2}^{z_{n}+h / 2} E_{z 1}(z, \omega) d z\right. \\
& \left.+\int_{z_{n}-h / 2-\tau}^{z_{n}-h / 2} E_{z 2}(z, \omega) d z\right) \\
= & \frac{\bar{E}_{z 1}\left(z_{n}, \omega\right) h+\bar{E}_{z 2}\left(z_{n}, \omega\right) \tau}{\tau+h},
\end{aligned}
$$

represents the average field integrated over one lamination width and one crack width. Thus, the surface impedances should be defined as

$$
\begin{aligned}
\overline{\mathcal{R}}_{z}(\omega, \eta) & =\left.\frac{\sum_{n} \bar{E}_{z}\left(z_{n}, \omega, \eta\right) e^{-j(\omega / c) z_{n}}}{\sum_{n} \bar{H}_{x}\left(z_{n}, \omega, \eta\right) e^{-j(\omega / c) z_{n}}}\right|_{y=b} \\
& =\left.\frac{\bar{E}_{z}(z=0, \omega, \eta)}{\bar{H}_{x}(z=0, \omega, \eta)}\right|_{y=b},
\end{aligned}
$$

provided $\left.\frac{\bar{E}_{z}\left(z_{n}, \omega, \eta\right)}{\bar{H}_{x}\left(z_{n}, \omega, \eta\right)}\right|_{y=b}$ does not depend on $z_{n}$.

Before calculating the surface impedance for the laminated structures some simplifications are in order. Using Eq. (43), one can write

$$
\overline{\mathcal{R}}_{z}(\omega, \eta)=\left.\frac{\bar{E}_{z 1} h+\bar{E}_{z 2} \tau}{\bar{H}_{x 1} h+\bar{H}_{x 2} \tau}\right|_{y=b} .
$$

As will be discussed at the end of Sec. II D 1, at the pipe wall

$$
\left.\left.\bar{H}_{x 1}\right|_{y=b} \approx \bar{H}_{x 2}\right|_{y=b} .
$$

Therefore

$$
\overline{\mathcal{R}}_{z}=\frac{\mathcal{R}_{1 z} h+\mathcal{R}_{2 z} \tau}{h+\tau},
$$

where $\mathcal{R}_{1 z}=\left.\frac{\bar{E}_{z 1}}{\bar{H}_{x 1}}\right|_{y=b}$ and $\mathcal{R}_{2 z}=\left.\frac{\bar{E}_{z 2}}{\bar{H}_{x 2}}\right|_{y=b}$ represent the longitudinal surface impedance in the crack and in the lamination, respectively.

Similarly, the horizontal surface impedance is

$$
\overline{\mathcal{R}}_{x}(\omega)=-\left.\frac{\bar{E}_{x 1} h+\bar{E}_{x 2} \tau}{\bar{H}_{z 1} h+\bar{H}_{z 2} \tau}\right|_{y=b} .
$$

Since $\bar{H}_{z 1}, \bar{E}_{x 1} \approx 0$, as will be discussed at the end of Sec. II D 1 ,

$$
\overline{\mathcal{R}}_{x}(\omega) \approx-\left.\frac{\bar{E}_{x 2}}{\bar{H}_{z 2}}\right|_{y=b} \approx \mathcal{R}_{2 x} .
$$

\section{Surface impedance}

In order to calculate the surface impedance, focus on the regions 1 and 2 shown in Fig. 3, i.e. $y>b$, and choose the middle of the crack as the origin $(z=0)$, also shown in the figure. Assume that the field dependence along the $z$ direction can be described by

$$
\begin{aligned}
& \frac{\partial^{2} E_{z}}{\partial z^{2}}=g_{1,2}^{2} E_{z} \\
& \frac{\partial^{2} H_{z}}{\partial z^{2}}=g_{1,2}^{2} H_{z},
\end{aligned}
$$

where the subscripts 1 and 2 correspond to the crack and lamination, respectively. The field equation is 


$$
\left(\frac{\partial^{2}}{\partial y^{2}}-\eta^{2}+g_{1,2}^{2}+\omega^{2} \mu \epsilon\right)\left(\begin{array}{c}
E_{z} \\
H_{z}
\end{array}\right)=0
$$

Region 1.-Denoting $q^{2}=\omega^{2} \mu_{1} \epsilon_{1}+g_{1}^{2}-\eta^{2}$, the solution of Eq. (52) inside the crack is

$$
\begin{gathered}
E_{z}=A_{1} \cosh \left(g_{1} z\right)(\sin q y+\alpha \cos q y) \cos \eta x \\
Z_{0} H_{z}=B_{1} \sinh \left(g_{1} z\right)(\alpha \sin q y-\cos q y) \sin \eta x .
\end{gathered}
$$

The transverse fields can be determined from the longitudinal fields [18], thus

$$
\begin{aligned}
\left(\omega^{2} \mu_{1} \epsilon_{1}+g_{1}^{2}\right) E_{x}= & \left(-A_{1} \eta g_{1}-B_{1} j \omega q \frac{\mu_{1 r}}{c}\right) \sinh \left(g_{1} z\right) \\
& \times(\sin q y+\alpha \cos q y) \sin \eta x \\
\left(\omega^{2} \mu_{1} \epsilon_{1}+g_{1}^{2}\right) Z_{0} H_{x}= & \left(-A_{1} j \omega q \frac{\epsilon_{1 r}}{c}+B_{1} \eta g_{1}\right) \cosh \left(g_{1} z\right) \\
& \times(\alpha \sin q y-\cos q y) \cos \eta x \\
\left(\omega^{2} \mu_{1} \epsilon_{1}+g_{1}^{2}\right) E_{y}= & \left(-A_{1} q g_{1}+B_{1} j \omega \eta \frac{\mu_{1 r}}{c}\right) \sinh \left(g_{1} z\right) \\
& \times(\alpha \sin q y-\cos q y) \cos \eta x \\
\left(\omega^{2} \mu_{1} \epsilon_{1}+g_{1}^{2}\right) Z_{0} H_{y}= & \left(A_{1} j \omega \eta \frac{\epsilon_{1 r}}{c}+B_{1} q g_{1}\right) \cosh \left(g_{1} z\right) \\
& \times(\sin q y+\alpha \cos q y) \sin \eta x .
\end{aligned}
$$

For the sake of simplicity, we assume that the crack terminates at $y=d$ in an ideal conductor. The condition $\left.E_{z}\right|_{y=d}=0$ implies

$$
\alpha=-\tan (q d) .
$$

The same constant $\alpha$ appears in the expression of all field components as written in Eqs. (54) and (56)-(58). This is a consequence of the condition $\left.H_{y}\right|_{y=d}=0 . E_{y} \propto \sinh \left(g_{1} z\right)$, since the current $J_{y}$ in the laminations along the crack goes in the opposite direction on the two sides of the crack. The dependence of the other field components on the sign of $z$ follows from the Maxwell equations.

Region 2.-Analogously, inside the lamination and close to the crack-lamination boundary

$$
\begin{aligned}
E_{z}=A_{2} e^{-g_{2}|z|}(\sin q y+\alpha \cos q y) \cos \eta x & \\
H_{z}=B_{2} e^{-g_{2}|z|} & \operatorname{ggn}(z)(\alpha \sin q y-\cos q y) \sin \eta x \\
\left(\omega^{2} \mu_{2} \epsilon_{2}+g_{2}^{2}\right) E_{x}= & \left(A_{2} \eta g_{2}-B_{2} j \omega q \frac{\mu_{2 r}}{c}\right) e^{-g_{2}|z|} \operatorname{sgn}(z) \\
& \times(\sin q y+\alpha \cos q y) \sin \eta x
\end{aligned}
$$

$$
\begin{aligned}
\left(\omega^{2} \mu_{2} \epsilon_{2}+g_{2}^{2}\right) Z_{0} H_{x}= & \left(-A_{2} j \omega q \frac{\epsilon_{2 r}}{c}-B_{2} \eta g_{2}\right) e^{-g_{2}|z|} \\
& \times(\alpha \sin q y-\cos q y) \cos \eta x \\
\left(\omega^{2} \mu_{2} \epsilon_{2}+g_{2}^{2}\right) E_{y}= & \left(A_{2} q g_{2}+B_{2} j \omega \eta \frac{\mu_{2 r}}{c}\right) e^{-g_{2}|z|} \operatorname{sgn}(z) \\
& \times(\alpha \sin q y-\cos q y) \cos \eta x \\
\left(\omega^{2} \mu_{2} \epsilon_{2}+g_{2}^{2}\right) Z_{0} H_{y}= & \left(A_{2} j \omega \eta \frac{\epsilon_{2 r}}{c}-B_{2} q g_{2}\right) e^{-g_{2}|z|} \\
& \times(\sin q y+\alpha \cos q y) \sin \eta x
\end{aligned}
$$

Only the exponentially decaying terms proportional to $e^{-g_{2}|z|}$ describe the fields in the laminations since it is assumed that the skin penetration depth is much less than the lamination thickness $\delta_{2} \ll \tau$. The continuity of the fields at $z= \pm h / 2$ implies

$$
q^{2}=\omega^{2} \mu_{2} \epsilon_{2}+g_{2}^{2}-\eta^{2}=\omega^{2} \mu_{1} \epsilon_{1}+g_{1}^{2}-\eta^{2} .
$$

Finding $g_{1}$ turns out to be enough for the calculation of the surface impedance. Since an exact solution requires substantial numerical effort, requiring field matching at the crack-lamination and chamber-wall boundaries, we take advantage of the high conductivity $\sigma_{2}$ in the laminations to provide an approximate solution. Note that if the laminations were ideal conductors then $E_{y}, E_{x},\left.H_{z}\right|_{z= \pm h / 2}=0$. This implies either

$$
g_{1}\left(\sigma_{2} \rightarrow \infty\right) \rightarrow 0
$$

or

$$
A_{1}\left(\sigma_{2} \rightarrow \infty\right) \rightarrow 0
$$

and

$$
B_{1}\left(\sigma_{2} \rightarrow \infty\right) \rightarrow 0
$$

The latter case yields a solution with a small electric field $E_{z}$ for large $\sigma_{2}$, thus giving a small contribution to the surface impedance. So, that case will be neglected to look for solutions which fulfill Eq. (67).

By imposing the continuity of $E_{x}, E_{y}, H_{x}$, and $H_{y}$ at $z=$ $h / 2$, one gets

$$
\begin{aligned}
\frac{A_{1}}{A_{2}} & =-\frac{\left(\eta g_{2}-\frac{B_{2}}{A_{2}} j \omega q \frac{\mu_{2 r}}{c}\right) e^{-g_{2} h / 2}}{\left(\eta g_{1}+\frac{B_{1}}{A_{1}} j \omega q \frac{\mu_{1 r}}{c}\right) \sinh \left(g_{1} h / 2\right)} \\
& =-\frac{\left(q g_{2}+\frac{B_{2}}{A_{2}} j \omega \eta \frac{\mu_{2 r}}{c}\right) e^{-g_{2} h / 2}}{\left(q g_{1}-\frac{B_{1}}{A_{1}} j \omega \eta \frac{\mu_{1 r}}{c}\right) \sinh \left(g_{1} h / 2\right)} \\
& =\frac{\left(j \omega q \frac{\epsilon_{2 r}}{c}+\frac{B_{2}}{A_{2}} \eta g_{2}\right) e^{-g_{2} h / 2}}{\left(j \omega q \frac{\epsilon_{1 r}}{c}-\frac{B_{1}}{A_{1}} \eta g_{1}\right) \cosh \left(g_{1} h / 2\right)} \\
& =\frac{\left(j \omega \eta \frac{\epsilon_{2 r}}{c}-\frac{B_{2}}{A_{2}} q g_{2}\right) e^{-g_{2} h / 2}}{\left(j \omega \eta \frac{\epsilon_{1 r}}{c}+\frac{B_{1}}{A_{1}} q g_{1}\right) \cosh \left(g_{1} h / 2\right)} .
\end{aligned}
$$


Equations (70) and (73) yield

$$
\begin{aligned}
& \frac{B_{2}}{A_{2}}=-\frac{B_{1}}{A_{1}} \frac{\mu_{1} g_{2}}{\mu_{2} g_{1}} \\
& \frac{B_{2}}{A_{2}}=-\frac{B_{1}}{A_{1}} \frac{\epsilon_{2} g_{1}}{\epsilon_{1} g_{2}} .
\end{aligned}
$$

If $B_{1} \neq 0$ and $B_{2} \neq 0$, Eqs. (74) and (75) together with Eq. (66) imply

$$
\begin{gathered}
g_{1}^{2}=-\omega^{2} \epsilon_{1} \mu_{1}=-\frac{\omega^{2}}{c^{2}} \epsilon_{1 r}, \\
g_{2}^{2}=-\omega^{2} \epsilon_{2} \mu_{2} .
\end{gathered}
$$

One can immediately see that this solution is not in agreement with the requirement of Eq. (67). If $B_{1}=0$ and $B_{2}=0$ then it follows from Eqs. (71) and (72) that

$$
\frac{g_{2} e^{-g_{2} h / 2}}{g_{1} \sinh \left(g_{1} h / 2\right)}=-\frac{\epsilon_{2} e^{-g_{2} h / 2}}{\epsilon_{1} \cosh \left(g_{1} h / 2\right)}
$$

or

$$
g_{1} \tanh \left(g_{1} h / 2\right)=-\frac{\epsilon_{1}}{\epsilon_{2}} g_{2}
$$

$g_{2}$ is large and represents the field decaying parameter inside the laminations. In the relevant frequency domain $g_{2}$ has the following form $[7,18]$ :

$$
g_{2} \approx \frac{1+j}{\delta_{2}}=\frac{(1+j) \sqrt{\omega \sigma_{2} \mu_{2}}}{\sqrt{2}} .
$$

Since $\epsilon_{2} \approx \frac{\sigma_{2}}{j \omega}$ and $g_{1} h \ll 1$,

$$
g_{1}^{2}=-\frac{2 j g_{2} \epsilon_{1} \omega}{h \sigma_{2}}=(1-j) \omega^{2} \epsilon_{1} \mu_{1} \frac{\mu_{2}}{\mu_{1}} \frac{\delta}{h} .
$$

This solution for $g_{1}$ obeys Eq. (67) and is the same as the one given by $\mathrm{Ng}$ in [7] where only the $\eta=0$ case was considered. However, note that while $g_{1}$ here and in Ref. [7] are the same, the surface impedance is significantly different.

The longitudinal surface impedance of the crack follows from Eqs. (53) and (56):

$$
\frac{\mathcal{R}_{1 z}(\eta, \omega)}{Z_{0}}=j \frac{q^{2}+\eta^{2}}{q \frac{\omega}{c} \epsilon_{1 r}} \tan q(d-b),
$$

where

$$
q^{2}=\omega^{2} \epsilon_{1} \mu_{1}\left[\left(1+(1-j) \frac{\mu_{2}}{\mu_{1}} \frac{\delta}{h}\right]-\eta^{2} .\right.
$$

The difference between this solution and that in Ref. [7] is the presence of the $\eta^{2}$ term in Eq. (83).
The lamination longitudinal and horizontal surface impedances are

$$
\mathcal{R}_{2 z} \approx \frac{1+j}{\delta_{2} \sigma_{2}}
$$

and

$$
\mathcal{R}_{2 x} \approx \frac{1+j}{\delta_{2} \sigma_{2}},
$$

which are equal to the usual metal-vacuum boundary surface impedance. The surface impedances $\mathcal{R}_{z}$ and $\mathcal{R}_{x}$ can be simply obtained now by employing Eqs. (47) and (49). Note that, unlike the resistive-wall case, the surface impedance is $\eta$ dependent. This turns out to have a significant effect on the impedance.

We now justify the approximations made in Eqs. (46) and (49).

Justification of Eq. (46).- Since $\tau \gg \delta_{2}$, away from the crack and at the lamination-chamber boundary (i.e. $y=+0+b$ ), the electromagnetic field decays along the vertical direction as

$$
\frac{\partial^{2} H_{x 2}}{\partial y^{2}} \approx g_{2}^{2} H_{x 2}
$$

which implies

$$
\left(\frac{\partial^{2}}{\partial z^{2}}-\eta^{2}+g_{2}^{2}+\omega^{2} \epsilon_{2} \mu_{2}\right) H_{x 2}=0 .
$$

Thus, $H_{x 2} \propto \cos (q z)$ away from the crack-lamination boundary. Since $q \sim \eta \sim \frac{1}{b}$ implies $q \tau \ll 1$, to a good approximation $H_{x 2}$ is constant along the $z$ direction in this region. The next region we focus on is the lamination close to both chamber and crack boundary. Inside the chamber (i.e. $y=-0+b$ ), the electric field component becomes very small $E_{y}(z \rightarrow \pm h / 2) \rightarrow \mathcal{O}\left(\sigma_{2}^{-(1 / 2)}\right)$ since it goes from being perpendicular to parallel to a metallic surface. This implies that the variation of $H_{x 2}$ with $z$ decreases in this region, because $\partial_{z} H_{x 2}=j \omega \epsilon_{0} E_{y}+$ $\partial_{x} H_{z 2}$. So far it has been argued that $H_{x 2}$ is nearly constant at the lamination-chamber boundary. In the crack, since $g_{1} h \ll 1, H_{x 1}$ is also approximately constant along $z$ direction. The continuity of $H_{x}$ at $z= \pm h / 2$ and $y=b$ implies $\left.\left.\bar{H}_{x 1}\right|_{y=b} \approx \bar{H}_{x 2}\right|_{y=b}$.

Justification of Eq. (49).- Since $H_{z 1}, E_{x 1} \propto \sinh \left(g_{1} z\right)$, their averaged value over the crack width is zero.

\section{Coupling impedance}

Using the surface impedance calculated in the previous section, the impedance of a laminated chamber is obtained by employing Eqs. (19), (21), and (23). Here we illustrate an example specific to the Fermilab Booster F-magnets, which have iron laminations. The chamber parameters are given in the caption of Fig. 4.

The longitudinal impedance is shown in Figs. 4(a) and 4(b). It has a very different behavior than a resistive-wall 


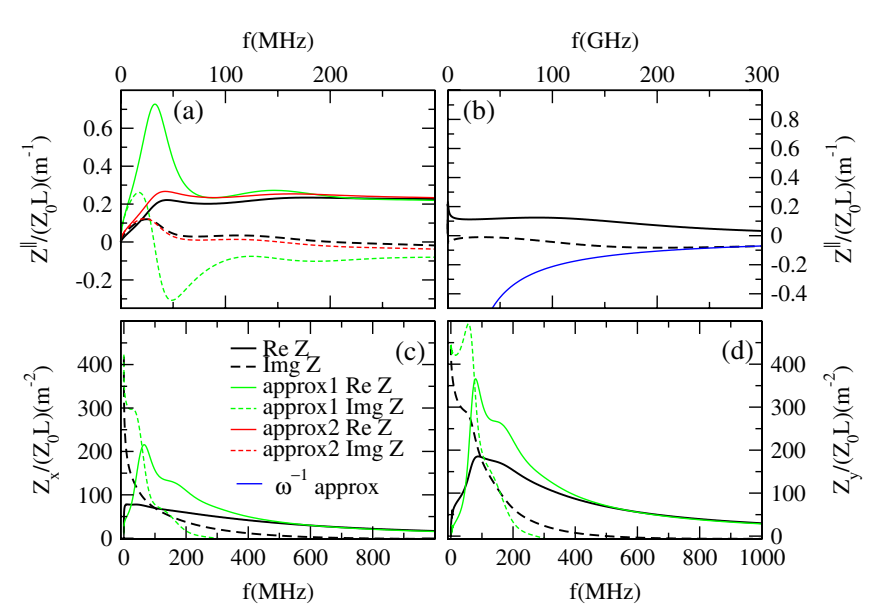

FIG. 4. Coupling impedances of a parallel-plane laminated chamber. We take $b=2.1 \mathrm{~cm}, \quad d=15.24 \mathrm{~cm}, \quad h=$ $9.52 \times 10^{-4} \mathrm{~cm}, \tau=6.35 \times 10^{-2} \mathrm{~cm}, \epsilon_{1 r}=4.75, \mu_{2 r}=100$, and $\sigma_{2}=0.5 \times 10^{7}(\Omega \mathrm{m})^{-1}$ (iron), parameters specific to the Fermilab Booster F-magnets. The real (imaginary) part is plotted with full (dashed) lines. The black lines show the full impedance calculation done by employing Eqs. (19), (21), and (23). Different approximations are also shown. The green lines are calculated by neglecting the $\eta$ dependence of the surface impedance, i.e., by making the approximation $\mathcal{R}_{z}(\eta) \approx \mathcal{R}_{z}(\eta=0)$ and employing Eqs. (35), (38), and (39). (a) Low frequency and (b) high frequency longitudinal impedance. The red lines are calculated by considering the full $\eta$ dependence of the surface impedance and by neglecting the terms proportional to the surface impedance in the denominator of Eq. (19), i.e., by employing Eq. (32). (c) Horizontal transverse impedance. (d) Vertical transverse impedance. The impedance is about 2 orders of magnitude larger than 1 corresponding to a resistivewall pipe made from the same material as the laminations. Note that neglecting the $\eta$ dependence in the surface impedance yields values that are too large for the coupling impedance.

impedance. At low frequencies, up to $30-40 \mathrm{MHz}$, it increases with increasing frequency, then in the interval 50-200 MHz it displays two broad and weakly developed peaks. A $\omega^{-(1 / 4)}$ behavior [6,7] follows up to $6 \mathrm{GHz}$. A third broad peak can be observed in the interval $20-80 \mathrm{GHz}$ and then the expected $\omega^{-1}$ behavior sets in at frequencies higher than $200 \mathrm{GHz}$.

The two commonly used approximations for the resistive-wall impedance, namely neglecting the $\mathcal{R}_{z}$ dependence on the horizontal wave-vector $\eta$ and neglecting the terms proportional to $\mathcal{R}_{z}$ in the denominator of Eq. (19), do not hold for laminated structures. The former was assumed by $\mathrm{Ng}$ [7] while the latter was implicitly assumed in Refs. [5,6] where the simple relation given by Eq. (35) between the surface impedance and the longitudinal coupling impedance was considered. The approximation Eq. (35) with $\mathcal{R}_{z}(\eta) \approx \mathcal{R}_{z}(\eta=0)$, valid for metallic pipes, is also plotted in Fig. 4(a). As one can see, Eq. (35) yields a much larger impedance for frequencies below $500 \mathrm{MHz}$.
In order to understand the importance of the $\eta$ dependence of $\mathcal{R}_{z}$ we will neglect for now the terms proportional to $\mathcal{R}_{z}$ in the denominator of Eq. (19) and will compare Eq. (32) versus Eq. (35) with $\mathcal{R}_{z}(\eta) \approx \mathcal{R}_{z}(\eta=0)$. In the latter case, one gets at low frequencies $q^{2} \approx(1-j) \times$ $\omega^{2} \epsilon_{1} \mu_{2} \frac{\delta}{h}, R_{z} \approx \frac{\mathcal{R}_{12} h}{h+\tau}=j \frac{q^{2}}{\epsilon_{1}}(d-b)$, and

$$
Z^{\|}=\frac{1}{2 \pi b} \frac{1+j}{\sigma_{2} \delta_{2}} \frac{2(d-b)}{h+\tau} .
$$

When the $\eta$ dependence is considered, at low frequency $q^{2}=-\eta^{2}$, the impedance is

$Z^{\|}=\frac{2}{2 \pi(h+\tau)} \frac{1+j}{\sigma_{2} \delta_{2}} \int_{0}^{\infty} d \eta \operatorname{sech}^{2} \eta b \frac{\tanh \eta(d-b)}{\eta}$.

In the case $\frac{d-b}{b} \ll 1$, the integral in Eq. (89) is $\frac{d-b}{b}$ and the impedance has the same form as in Eq. (88). However, when $\frac{d-b}{b} \gg 1$, as in our example, the integral is

$$
\begin{aligned}
& \int_{0}^{\infty} d \eta \operatorname{sech}^{2} \eta b \frac{\tanh \eta(d-b)}{\eta} \\
& \quad \approx \int_{0}^{b} d \eta \frac{\tanh \eta(d-b)}{\eta} \\
& \quad \approx \int_{0}^{1 /(d-b)} d \eta \frac{\eta(d-b)}{\eta}+\int_{1 /(d-b)}^{b} d \eta \frac{1}{\eta} \\
& \quad=1+\ln \frac{d-b}{b} \approx \ln \frac{d-b}{b},
\end{aligned}
$$

and

$$
Z^{\|}=\frac{1}{2 \pi b} \frac{1+j}{\sigma_{2} \delta_{2}} \frac{2}{h+\tau} b \ln \frac{(d-b)}{b} .
$$

Thus, at low frequencies and to first order in $\mathcal{R}_{z}$, the impedance increases logarithmically with $d-b$ when $d$ is large, similarly to the case of circular geometry (see Sec. III and [7]) and not linearly as one would wrongly infer if the $\eta$ dependence were neglected.

Besides considering the $\eta$ dependence of $\mathcal{R}_{z}$, taking into account terms proportional to $\mathcal{R}_{z}$ in the denominator of Eq. (19) is also important. The reason, as discussed in Sec. IIC, is that $\mathcal{R}_{z}$ is large when the laminations are present, being enhanced roughly by a factor of $\frac{2 b}{h+\tau} \ln \frac{d-b}{b}$ (which in our example is $\approx 115$ ) compared to the resistivewall case. The approximation given by Eq. (32), shown in red in Fig. 4(a), although taking into account the $\eta$ dependence of $\mathcal{R}_{z}$, neglects the $\mathcal{R}_{z}$ terms in the denominator of Eq. (19). The low frequency behavior is given by Eq. (89) and has a $\sqrt{\omega}$ behavior. However, this approximation differs from the full calculation (black) which yields a smaller impedance.

The horizontal and the vertical impedances are shown in Figs. 4(c) and 4(d), respectively. Compared to the resistivewall impedances for an iron chamber with the same dimensions (Fig. 1), they are about 2 orders of magnitude larger. For frequencies larger than $15 \mathrm{MHz}$, the vertical 
impedance is about 2 times larger than the horizontal one, a behavior characteristic of parallel-plane geometry [16]. The large frequency asymptotic behavior is $\omega^{-5 / 4}$. Note that the $\eta$ dependence of $\mathcal{R}_{z}$ is also important for the transverse impedances. Neglecting it would yield a much larger value for the impedance, as shown by the green lines where Eqs. (38) and (39), respectively, are employed.

\section{Wake functions}

The wake functions calculated via Eqs. (4) and (5) from the impedances shown in Fig. 4 are displayed in Fig. 5. For comparison different approximations and the resistive-wall impedance of an iron chamber with the same dimensions are also illustrated.

The longitudinal wake, shown in black in Fig. 5(a), is repulsive at distances shorter than $0.2 \mathrm{~m}$, while it becomes attractive at somewhat larger distances, with a maximum around $|z| \approx 0.4 \mathrm{~m}$. It decreases rapidly to zero at large distances. In the attractive region, at distances on the order of meters (which is usually of the same order as the bunch length in typical machines) it is much larger than the resistive-wall impedance (blue). Note that the approximation based on Eq. (35), which neglects the $\eta$ dependence of

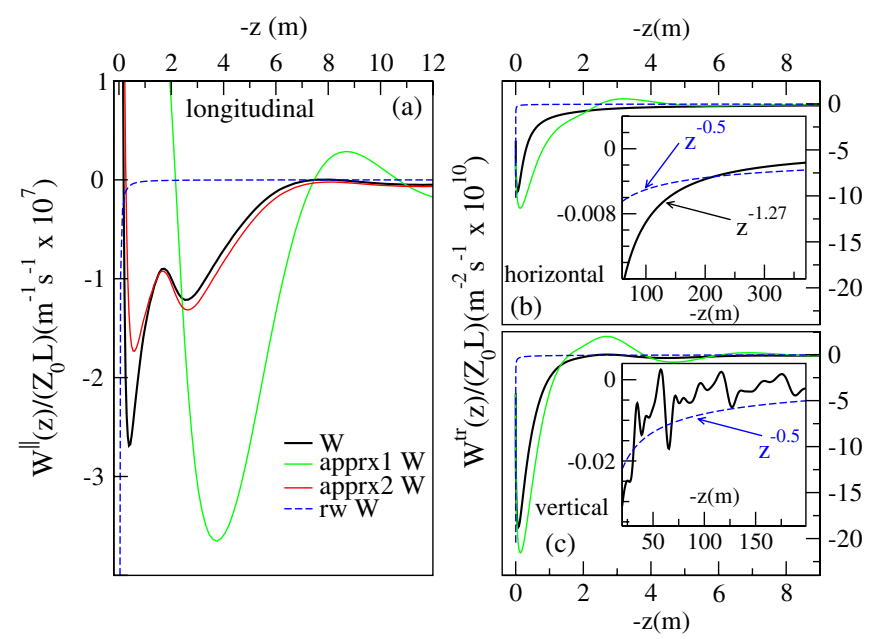

FIG. 5. Wake functions for a laminated chamber with parallelplane geometry (black). The parameters are the same as in Fig. 4. (a) Longitudinal wake function versus distance $|z|$. The wake is repulsive up to $|z| \approx 0.2 \mathrm{~m}$, then becomes attractive with a maximum around $|z| \approx 0.4 \mathrm{~m}$. The longitudinal wake is much larger than the resistive-wall case for an iron chamber (blue). The approximations which neglect the $\eta$ dependence of $\mathcal{R}_{z}$ (green) and the term proportional to $\mathcal{R}_{z}$ in the denominator of Eq. (19) (red) yield significantly different results. (b) and (c) The horizontal (b) and vertical (c) wake functions. Compared to the resistive-wall wake (blue) they are much larger at distance of order of meters. However, they decay faster with increasing $|z|$. The horizontal wake becomes smaller than the resistive-wall wake at $|z|>200 \mathrm{~m}$, while the vertical one becomes smaller at $|z|>30 \mathrm{~m}$; see the inset. Neglecting the $\eta$ dependence of $\mathcal{R}_{z}$ (green) yields larger transverse wakes.
$\mathcal{R}_{z}$ (green), yields a larger wake, repulsive for $|z|<2 \mathrm{~m}$. Neglecting the $\mathcal{R}_{z}$ terms in the denominators of Eq. (19) (red) yields a smaller impedance at distances of the order of $1 \mathrm{~m}$.

The horizontal and vertical wakes are shown in Figs. 5(b) and 5(c), respectively. The vertical wake is about 2 times larger than the horizontal one for distances of order of meters. In the same region the wakes are much larger than the corresponding resistive-wall wakes (blue), but decay faster at large $|z|$. Neglecting the $\eta$ dependence of $\mathcal{R}_{z}$ (green) has a noticeable effect, yielding larger transverse wakes. The wiggling behavior of the vertical wake, seen in the inset of Fig. 5(c), is caused by the term proportional to $\mathcal{R}_{z} \frac{k}{\eta}$ in the denominator of Eq. (21). This shows again that the higher-order terms in the surface impedance cannot be neglected in the coupling impedance calculation for laminated structures.

\section{CIRCULAR CHAMBER WITH LAMINATIONS}

\section{A. Wake functions}

Up to the first order in the beam displacement the momentum kick due to wakefields in circular chambers is given by Eqs. (1)-(3) with the quadrupole term, i.e., the term proportional with the trailing particle displacement, zero [8]. We will append the subscript 0 to the longitudinal wake notation, i.e. $W^{\|} \equiv W_{0}^{\|}$to indicate that it corresponds to the angular number $m=0$. Because of the symmetry the kicks along the $x$ and $y$ directions are described by the same function $W_{x}^{\perp}=W_{y}^{\perp} \equiv W_{1}^{\perp}$ and correspond to $m=1$. The wakes and the impedances are related via Fourier transforms such as in Eqs. (4) and (5).

\section{B. Impedance}

As for the parallel-plane geometry, it is desirable to first derive equations which describe the coupling impedance as a function of the surface impedance.

The Maxwell equations are solved in cylindrical coordinates. The charge and current decomposition are

$$
\begin{gathered}
\rho(r, \theta, z, t)=\sum_{m=0}^{\infty} \rho_{m} \delta(r-b) e^{j(\omega t-k z)} \\
\vec{J}(r, \theta, z, t)=\rho(r, \theta, z, t) c \hat{z}
\end{gathered}
$$

where

$$
\begin{gathered}
\rho_{m}=\frac{I_{m}}{\pi b^{m+1}\left(1+\delta_{m 0}\right)}, \\
I_{m}=\rho b^{m} .
\end{gathered}
$$

The field decomposition reads

$$
\left[E_{r}(r, \theta), E_{z}(r, \theta), H_{\theta}(r, \theta)\right]=\sum_{m=0}^{\infty}\left(E_{r m}, E_{z m}, H_{\theta m}\right) \cos (m \theta)
$$




$$
\left[E_{\theta}(r, \theta), H_{r}(r, \theta), H_{z}(r, \theta)\right]=\sum_{m=0}^{\infty}\left(E_{\theta m}, H_{r m}, H_{z m}\right) \sin (m \theta) .
$$

Note that for $m=0$ the field components $E_{\theta}(r, \theta)$, $H_{r}(r, \theta), H_{z}(r, \theta)$ are zero.

The solution of the electromagnetic field inside a round chamber has been calculated by numerous authors, see, e.g., Refs. [7,8]. Inside the chamber, i.e., for $r<b$,

$$
E_{z 0}=A_{0},
$$

for $m=0$ and

$$
\begin{gathered}
E_{z m}=A_{m} r^{m} \\
Z_{0} H_{z m}=-A_{m} r^{m},
\end{gathered}
$$

for $m \geq 1$.

The constants $A_{0}$ and $A_{m}$ are determined from the boundary conditions at the pipe walls. They can also be expressed as functions of surface impedances

$$
\begin{gathered}
\mathcal{R}_{z m}=-\left.\frac{E_{z m}}{H_{\theta m}}\right|_{r=b} \\
\mathcal{R}_{\theta m}=\left.\frac{E_{\theta}}{H_{z m}}\right|_{r=b},
\end{gathered}
$$

with

$$
\begin{gathered}
A_{0}=\frac{-\frac{\rho_{1} \mathcal{R}_{z 0}}{Z_{0} 2 \pi \epsilon_{0} b}}{1+j \frac{\mathcal{R}_{z 0}}{Z_{0}} \frac{k b}{2}}, \quad m=0 \\
A_{m}=\frac{-\frac{I_{m} \mathcal{R}_{z m}}{Z_{0} \pi \epsilon_{0} b^{2 m+1}}}{1+j \frac{\mathcal{R}_{z m}}{Z_{0}}\left(\frac{k b}{m+1}-\frac{m}{b k}\right)+\frac{\mathcal{R}_{z m} \mathcal{R}_{\theta m}}{Z_{0}^{2}}}, \quad m \geq 1 .
\end{gathered}
$$

The longitudinal coupling impedance as a function of surface impedance reads

$$
\begin{gathered}
Z_{0}^{\|}=-\frac{E_{z}}{c \rho_{1}}=\frac{\frac{\mathcal{R}_{z 0}}{2 \pi b}}{1+j \frac{\mathcal{R}_{z 0}}{Z_{0}} \frac{\mathrm{kb}}{2}}, \quad m=0 \\
Z_{m}^{\|}=-\frac{E_{z}}{c I_{m} r^{m}}=\frac{\frac{\mathcal{R}_{z m}}{\pi b^{2 m+1}}}{1+j \frac{\mathcal{R}_{z m}}{Z_{0}}\left(\frac{k b}{m+1}-\frac{m}{b k}\right)+\frac{\mathcal{R}_{z m} \mathcal{R}_{\theta m}}{Z_{0}^{2}}}, \quad m \geq 1,
\end{gathered}
$$

while from Panofsky-Wenzel theorem the transverse impedance is

$$
Z_{m}^{\perp}=\frac{c}{\omega} Z_{m}^{\|}=\frac{\frac{c \mathcal{R}_{z m}}{\omega \pi b^{2 m+1}}}{1+j \frac{\mathcal{R}_{z m}}{Z_{0}}\left(\frac{k b}{m+1}-\frac{m}{b k}\right)+\frac{\mathcal{R}_{z m} \mathcal{R}_{\theta m}}{Z_{0}^{2}}}, \quad m \geq 1 .
$$

\section{Resistive-wall impedance}

The equation for longitudinal field inside a cylindrical metallic pipe is

$$
\frac{1}{r} \frac{\partial}{\partial r}\left(r \frac{\partial}{\partial r}\right)\left(\begin{array}{c}
E_{z} \\
H_{z}
\end{array}\right)=\left(\frac{m^{2}}{r}+\lambda_{c}^{2}+p^{2}\right)\left(\begin{array}{c}
E_{z} \\
H_{z}
\end{array}\right),
$$

where $\lambda_{c}^{2}=j \omega \sigma \mu=j k Z_{0} \sigma \mu_{r}$ and $p^{2}=k^{2}\left(1-\epsilon_{r} \mu_{r}\right)$.

The transverse fields are derived from the longitudinal ones, see, e.g., Jackson [18].

We treat the $m=0$ case first. The solution is

$$
\begin{gathered}
E_{z}=A_{0} \frac{K_{0}(\lambda r)}{K_{0}(\lambda b)} \quad E_{r}=A_{0} j \frac{k}{\lambda} \frac{K_{0}^{\prime}(\lambda r)}{K_{0}(\lambda b)} \\
Z_{0} H_{\theta}=A_{0} \frac{j k+Z_{0} \sigma}{\lambda} \frac{K_{0}^{\prime}(\lambda r)}{K_{0}(\lambda b)},
\end{gathered}
$$

where $\lambda^{2}=\lambda_{c}^{2}+p^{2}$ and $K_{0}$ is the modified Bessel function of the second kind and order zero. It follows that

$$
\frac{Z_{0}}{\mathcal{R}_{z 0}}=-\frac{j k+Z_{0} \sigma}{\lambda} \frac{K_{0}^{\prime}(\lambda b)}{K_{0}(\lambda b)} .
$$

For $m \geq 1$

$$
\begin{aligned}
E_{z} & =-Z_{0} H_{z}=A_{m} b^{m} \frac{K_{m}(\lambda r)}{K_{m}(\lambda b)} \\
E_{r} & =E_{\theta}=A_{m} b^{m} \frac{j k}{\lambda}\left(\frac{K_{m}^{\prime}(\lambda r)}{K_{m}(\lambda b)}-\frac{m}{\lambda r} \frac{K_{m}(\lambda r)}{K_{m}(\lambda b)}\right) \\
Z_{0} H_{\theta} & =A_{m} b^{m}\left(\frac{j k+Z_{0} \sigma}{\lambda} \frac{K_{m}^{\prime}(\lambda r)}{K_{m}(\lambda b)}-\frac{j k m}{\lambda^{2} r} \frac{K_{m}(\lambda r)}{K_{m}(\lambda b)}\right) \\
Z_{0} H_{r} & =-A_{m} b^{m}\left(\frac{j k}{\lambda} \frac{K_{m}^{\prime}(\lambda r)}{K_{m}(\lambda b)}-\frac{m}{\lambda r} \frac{j k+Z_{0} \sigma}{\lambda} \frac{K_{m}(\lambda r)}{K_{m}(\lambda b)}\right),
\end{aligned}
$$

where $K_{m}$ is the modified Bessel function of second kind and of order $m$. One gets

$$
\begin{array}{r}
\frac{Z_{0}}{\mathcal{R}_{z m}}=-\frac{j k+Z_{0} \sigma}{\lambda} \frac{K_{m}^{\prime}(\lambda b)}{K_{m}(\lambda b)}+\frac{j k m}{\lambda^{2} b}, \\
\frac{\mathcal{R}_{\theta m}}{Z_{0}}=-\frac{j k}{\lambda}\left(\frac{K_{m}^{\prime}(\lambda b)}{K_{m}(\lambda b)}-\frac{m}{\lambda b}\right) .
\end{array}
$$

Note that for the metallic round chamber, up to $\mathcal{O}\left(\sigma^{-1 / 2}\right)$,

$$
\mathcal{R}_{z 0} \approx \mathcal{R}_{z m} \approx \mathcal{R}_{\theta m} \approx \frac{\lambda_{c}}{\sigma}
$$

as is the case for the flat metallic chamber [see Eq. (31)]. In this approximation the surface impedance is independent of $m$. However, as we will see in Sec. III D, the $m$ dependence of the surface impedance is important in the calculation of the transverse impedance for a laminated circular chamber.

Since the surface impedance is small for metallic chambers, the terms proportional to it in the denominator of Eqs. (105)-(107) can be neglected. The commonly used approximation for impedance in circular metallic pipes is 


$$
\begin{aligned}
Z_{0}^{\|} & =\frac{\mathcal{R}_{z}}{2 \pi b}, \\
Z_{1}^{\perp} & =\frac{\mathcal{R}_{z}}{\pi k b^{3}},
\end{aligned}
$$

with $\mathcal{R}_{z}$ given by Eq. (114). Note that, compared to the horizontal and the vertical impedances for the flat metallic chamber, Eqs. (36) and (37), the circular transverse impedance is larger by factors of $\frac{24}{\pi^{2}}$ and $\frac{12}{\pi^{2}}$, respectively.

Another commonly encountered relation is given by

$$
Z_{1}^{\perp}=\frac{2 c}{\omega b^{2}} Z_{0}^{\|} .
$$

\section{Laminated structures}

Using the same justifications as for the flat chamber in Sec. II D, we have

$$
\overline{\mathcal{R}}_{z}=\frac{\mathcal{R}_{1 z} h+\mathcal{R}_{2 z} \tau}{h+\tau}
$$

and

$$
\overline{\mathcal{R}}_{\theta}(\omega) \approx \mathcal{R}_{2 \theta},
$$

where " 1 " denotes the crack region and " 2 " the region of the lamination.

\section{Surface impedance}

For large conductivity $\sigma_{2}$, the lamination surface impedances are given by

$$
\mathcal{R}_{2 z m} \approx \frac{1+j}{\delta_{2} \sigma_{2}},
$$

and

$$
\mathcal{R}_{2 \theta m} \approx \frac{1+j}{\delta_{2} \sigma_{2}}, \quad m \geq 1
$$

The next step is to calculate the crack surface impedance $\mathcal{R}_{1 z m}$. As for the flat chamber, we assume that the field dependence along the longitudinal direction is given by Eqs. (50) and (51). Thus, we have

$$
\left[\frac{1}{r} \frac{\partial}{\partial r}\left(r \frac{\partial}{\partial r}\right)-\frac{m^{2}}{r}+q^{2}\right]\left(\begin{array}{c}
E_{z} \\
H_{z}
\end{array}\right)=0
$$

with $q^{2}=\omega^{2} \epsilon_{1,2} \mu_{1,2}+g_{1,2}^{2}$.

Focusing on the $m=0$ case first, the fields inside the crack are given by

$$
\begin{gathered}
E_{z}=A_{1} \cosh \left(g_{1} z\right)\left[H_{0}^{(2)}(q r)+\alpha H_{0}^{(1)}(q r)\right] \\
E_{r}=-A_{1} \frac{g_{1}}{q} \sinh \left(g_{1} z\right)\left[H_{1}^{(2)}(q r)+\alpha H_{1}^{(1)}(q r)\right]
\end{gathered}
$$

$$
Z_{0} H_{\theta}=A_{1} \frac{j \omega \epsilon_{1 r}}{q c} \cosh \left(g_{1} z\right)\left[H_{1}^{(2)}(q r)+\alpha H_{1}^{(1)}(q r)\right],
$$

where $H_{n}^{(1),(2)}=J_{n} \pm j Y_{n}$ are Bessel functions of the third kind and order $n . J_{n}$ and $Y_{n}$ are Bessel functions of order $n$ and of the first and, respectively, the second kind. Inside the laminations

$$
\begin{gathered}
E_{z}=A_{2} e^{-g_{2}|z|}\left[H_{0}^{(2)}(q r)+\alpha H_{0}^{(1)}(q r)\right] \\
E_{r}=A_{2} e^{-g_{2}|z|} \operatorname{sgn}(z) \frac{g_{2}}{q}\left[H_{1}^{(2)}(q r)+\alpha H_{1}^{(1)}(q r)\right] \\
Z_{0} H_{\theta}=A_{2} \frac{j \omega \epsilon_{2 r}}{q c} e^{-g_{2}|z|}\left[H_{1}^{(2)}(q r)+\alpha H_{1}^{(1)}(q r)\right] .
\end{gathered}
$$

The condition $\left.E_{z}\right|_{r=d}=0$ implies

$$
\alpha=-\frac{H_{0}^{(2)}(q d)}{H_{0}^{(1)}(q d)} .
$$

The continuity of the transverse fields at $z= \pm \frac{h}{2}$ requires $\omega^{2} \epsilon_{1} \mu_{1}+g_{1}^{2}=\omega^{2} \epsilon_{2} \mu_{2}+g_{2}^{2}=q^{2}$ and

$$
g_{1} \tanh \left(g_{1} h / 2\right)=-\frac{\epsilon_{1}}{\epsilon_{2}} g_{2} \text {. }
$$

This gives

$$
g_{1}^{2}=-\frac{2 j g_{2} \epsilon_{1} \omega}{h \sigma_{2}}=(1-j) \omega^{2} \epsilon_{1} \mu_{1} \frac{\mu_{2}}{\mu_{1}} \frac{\delta}{h},
$$

where we assume $g_{2} \approx \frac{1+j}{\delta_{2}}=\left[(1+j) \sqrt{\omega \sigma_{2} \mu_{2}}\right] / \sqrt{2}$, as for the flat chamber.

The crack surface impedance is

$$
\frac{\mathcal{R}_{1 z 0}}{Z_{0}}=j \frac{q c}{\omega \epsilon_{r 1}} \frac{H_{0}^{(2)}(q b) H_{0}^{(1)}(q d)-H_{0}^{(1)}(q b) H_{0}^{(2)}(q d)}{H_{1}^{(2)}(q b) H_{0}^{(1)}(q d)-H_{1}^{(1)}(q b) H_{0}^{(2)}(q d)}
$$

$$
=j \frac{q c}{\omega \epsilon_{r 1}} \frac{J_{0}(q b) Y_{0}(q d)-Y_{0}(q b) J_{0}(q d)}{J_{1}(q b) Y_{0}(q d)-Y_{1}(q b) J_{0}(q d)} .
$$

This is the same result as in Ref. [6,7]. For $m \geq 1$ the fields in the crack are

$$
E_{z}=A_{1} \cosh \left(g_{1} z\right)\left[H_{m}^{(2)}(q r)+\alpha H_{m}^{(1)}(q r)\right] \cos (m \theta)
$$

$Z_{0} H_{z}=B_{1} \sinh \left(g_{1} z\right)\left[H_{m}^{(2)}(q r)+\beta H_{m}^{(1)}(q r)\right] \sin (m \theta)$

$$
\begin{aligned}
E_{\theta}= & {\left[-A_{1} \frac{g_{1} m}{q^{2} r}\left[H_{m}^{(2)}(q r)+\alpha H_{m}^{(1)}(q r)\right]+B_{1} \frac{j k \mu_{r 1}}{q}\right.} \\
& \left.\times\left[H_{m}^{\prime(2)}(q r)+\beta H_{m}^{\prime(1)}(q r)\right]\right] \sinh \left(g_{1} z\right) \sin (m \theta)
\end{aligned}
$$




$$
\begin{aligned}
Z_{0} H_{\theta}= & {\left[-A_{1} \frac{j k \epsilon_{r 1}}{q}\left[H_{m}^{\prime(2)}(q r)+\alpha H_{m}^{\prime(1)}(q r)\right]+B_{1} \frac{g_{1} m}{q^{2} r}\right.} \\
& \left.\times\left[H_{m}^{(2)}(q r)+\beta H_{m}^{(1)}(q r)\right]\right] \cosh \left(g_{1} z\right) \cos (m \theta) \\
E_{r}= & {\left[A_{1} \frac{g_{1}}{q}\left[H_{m}^{\prime(2)}(q r)+\alpha H_{m}^{\prime(1)}(q r)\right]-B_{1} \frac{j k m \mu_{r 1}}{q^{2} r}\right.} \\
& \left.\times\left[H_{m}^{(2)}(q r)+\beta H_{m}^{(1)}(q r)\right]\right] \sinh \left(g_{1} z\right) \cos (m \theta) \\
Z_{0} H_{r}= & {\left[-A_{1} \frac{j k m \epsilon_{r 1}}{q^{2} r}\left[H_{m}^{(2)}(q r)+\alpha H_{m}^{(1)}(q r)\right]+B_{1} \frac{g_{1}}{q}\right.} \\
& \left.\times\left[H_{m}^{\prime(2)}(q r)+\beta H_{m}^{\prime(1)}(q r)\right]\right] \cosh \left(g_{1} z\right) \sin (m \theta) .
\end{aligned}
$$

In the lamination we have

$$
\begin{aligned}
E_{z}= & A_{2} e^{-g_{2}|z|}\left[H_{m}^{(2)}(q r)+\alpha H_{m}^{(1)}(q r)\right] \cos (m \theta) \\
Z_{0} H_{z}= & B_{2} e^{-g_{2}|z|} \operatorname{sgn}(z)\left[H_{m}^{(2)}(q r)+\beta H_{m}^{(1)}(q r)\right] \sin (m \theta) \\
E_{\theta}= & {\left[A_{2} \frac{g_{2} m}{q^{2} r}\left[H_{m}^{(2)}(q r)+\alpha H_{m}^{(1)}(q r)\right]+B_{2} \frac{j k \mu_{r 2}}{q}\right.} \\
& \left.\times\left[H_{m}^{\prime(2)}(q r)+\beta H_{m}^{\prime(1)}(q r)\right]\right] e^{-g_{2}|z|} \operatorname{sgn}(z) \sin (m \theta) \\
Z_{0} H_{\theta}= & {\left[-A_{2} \frac{j k \epsilon_{r 2}}{q}\left[H_{m}^{\prime(2)}(q r)+\alpha H_{m}^{\prime(1)}(q r)\right]-B_{2} \frac{g_{2} m}{q^{2} r}\right.} \\
& \left.\times\left[H_{m}^{(2)}(q r)+\beta H_{m}^{(1)}(q r)\right]\right] e^{-g_{2}|z|} \cos (m \theta) \\
E_{r}= & {\left[-A_{2} \frac{g_{2}}{q}\left[H_{m}^{\prime(2)}(q r)+\alpha H_{m}^{\prime(1)}(q r)\right]-B_{2} \frac{j k m \mu_{r 2}}{q^{2} r}\right.} \\
\times & {\left.\left[H_{m}^{(2)}(q r)+\beta H_{m}^{(1)}(q r)\right]\right] e^{-g_{2}|z|} s g n(z) \cos (m \theta) } \\
& \left.\times\left[H_{m}^{\prime(2)}(q r)+\beta H_{m}^{\prime(1)}(q r)\right]\right] e^{-g_{2}|z|} \sin (m \theta) . \\
Z_{0} H_{r}= & {\left[-A_{2} \frac{j k m \epsilon_{r 2}}{q^{2} r}\left[H_{m}^{(2)}(q r)+\alpha H_{m}^{(1)}(q r)\right]-B_{2} \frac{g_{2}}{q}\right.} \\
& \\
&
\end{aligned}
$$

As for the flat chamber, the condition for an acceptable solution is $B_{1}=B_{2}=0 . \quad E_{z}=\left.0\right|_{r=d}$ implies $\alpha=$
$-\left\{\left[H_{m}^{(2)}(q d)\right] /\left[H_{m}^{(1)}(q d)\right]\right\}$. The continuity of the transverse fields at $z= \pm \frac{h}{2}$ implies the same Eq. (131) for $g_{1}$.

Consequently, the crack surface impedance reads

$$
\begin{aligned}
\frac{\mathcal{R}_{1 z m}}{Z_{0}} & =-j \frac{q c}{\omega \epsilon_{r 1}} \frac{H_{m}^{(2)}(q b) H_{m}^{(1)}(q d)-H_{m}^{(1)}(q b) H_{m}^{(2)}(q d)}{H_{m}^{\prime(2)}(q b) H_{m}^{(1)}(q d)-H_{m}^{\prime(1)}(q b) H_{m}^{(2)}(q d)} \\
& =-j \frac{q c}{\omega \epsilon_{r 1}} \frac{J_{m}(q b) Y_{m}(q d)-Y_{m}(q b) J_{m}(q d)}{J_{m}^{\prime}(q b) Y_{m}(q d)-Y_{m}^{\prime}(q b) J_{m}(q d)},
\end{aligned}
$$

where

$$
q^{2}=\omega^{2} \epsilon_{1} \mu_{1}\left[\left(1+(1-j) \frac{\mu_{2}}{\mu_{1}} \frac{\delta}{h}\right]\right.
$$

\section{Coupling impedance for round chamber and comparison with flat chamber}

In Fig. 6(a) we show the crack surface impedance for angular numbers $m=0$ and $m=1$. Note that they are significantly different, thus producing different longitudinal wakes, unlike the resistive-wall case. The low frequency behavior can be deduced by considering small- $x$ approximations of the Bessel functions, $J_{0}(x) \approx 1$, $Y_{0}(x) \approx \frac{2}{\pi}\left(\ln \frac{x}{2}+\gamma_{e}\right)$ with $\gamma_{e}=0.5772$ being the EulerMascheroni constant, $J_{1}(x) \approx \frac{1}{2} x$ and $Y_{1}(x) \approx-\frac{2}{\pi} \frac{1}{x}$. One gets

$\mathcal{R}_{1 z 0} \approx j \frac{q^{2}}{\omega \epsilon_{1}} b \ln \frac{d}{b} \approx \frac{2}{h} \frac{1+j}{\delta_{2} \sigma_{2}} b \ln \frac{d}{b}, \quad m=0$

and

$$
\begin{aligned}
\mathcal{R}_{1 z 1} & \approx j \frac{q^{2}}{\omega \epsilon_{1}} b \frac{d^{2}-b^{2}}{d^{2}+b^{2}} \approx \frac{2}{h} \frac{1+j}{\delta_{2} \sigma_{2}} b \frac{d^{2}-b^{2}}{d^{2}+b^{2}}, \\
m & =1 .
\end{aligned}
$$

Both $m=0$ and $m=1$ cases display resistive-wall-like behavior at small frequencies, i.e., the surface impedance is proportional to $\sqrt{\omega}$. However, the $m=1$ surface impedance has a much smaller proportionality factor, $\left(d^{2}-b^{2}\right) /\left(d^{2}+b^{2}\right)$ compared to $\ln \frac{d}{b}$. In our example the resistive-wall behavior of the surface impedance can be observed up to 10-15 MHz.

Since the $m=0$ and the $m=1$ surface impedances are different, the relation Eq. (117) between the longitudinal $Z_{0}^{\|}$and the transverse $Z_{1}^{\perp}$ impedances does not hold.

In Figs. 6(b) and 6(c) a comparison between the circular chamber impedances and those corresponding to a flat chamber with the same parameters is shown. In the circular chamber case, the longitudinal impedance has more strongly developed peaks around $40 \sim 50 \mathrm{MHz}$. For the comparison of the transverse impedances the flat chamber horizontal and vertical impedances are multiplied by the factors $\frac{24}{\pi^{2}}$ and $\frac{12}{\pi^{2}}$, respectively (see the discussion at the 
end of Sec. IIIC). Other significant differences in the transverse impedances of flat and round chambers can be noticed below $400 \mathrm{MHz}$.

\section{Wake functions for round chamber and comparison with flat chamber}

The wake functions for a round laminated chamber, obtained via Fourier transforms from the impedances shown in Fig. 6, are plotted in Fig. 7. For comparison, the wake functions corresponding to a flat laminated chamber with the same parameters are also shown.

Figure 7(a) displays the longitudinal wakes. Both circular (black) and flat (blue) longitudinal wakes are repulsive below $0.2 \mathrm{~m}$ and attractive in the range $0.2-5 \mathrm{~m}$. The flat chamber wake is larger around $0.4 \mathrm{~m}$ while the round chamber one is more strongly peaked around $2.6 \mathrm{~m}$. At $\approx 6 \mathrm{~m}$ the round chamber longitudinal wake has a repulsive peak, becoming attractive again at $\approx 7 \mathrm{~m}$, while the flat wake is smaller and attractive in this distance range. There is no significant difference between the longitudinal wakes at distances larger than $11 \mathrm{~m}$. However, they are already small in this region.

The transverse wakes are shown in Fig. 7(b). At distances of the order of a few meters, aside from the geometrically specific factors $\frac{24}{\pi^{2}}$ and $\frac{12}{\pi^{2}}$, the circular transverse wake is close to the horizontal and vertical parallel-plane

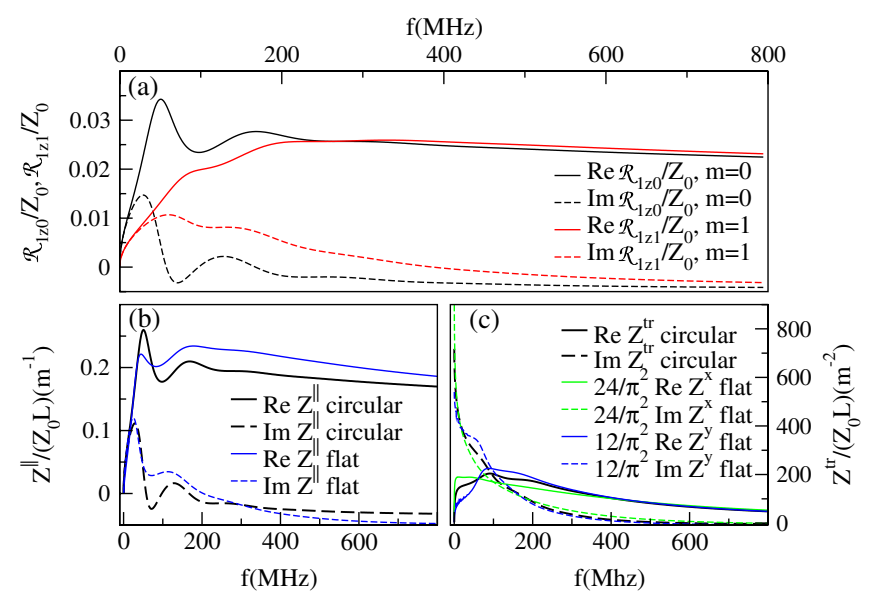

FIG. 6. The parameters for the round chamber with laminations are the same as for our flat chamber example, $b=2.1 \mathrm{~cm}$, $d=15.24 \mathrm{~cm}, \quad h=9.52 \times 10^{-4} \mathrm{~cm}, \quad \tau=6.35 \times 10^{-2} \mathrm{~cm}$, $\epsilon_{1 r}=4.75, \quad \mu_{2 r}=100, \quad$ and $\quad \sigma_{2}=0.5 \times 10^{7}(\Omega \mathrm{m})^{-1}$. (a) Crack surface impedance $\mathcal{R}_{1 z 0}$ ( $m=0$, black) and $\mathcal{R}_{1 z 1}$ ( $m=1$, red). At low frequency both cases display resistive-wall behavior proportional to $\sqrt{\omega}$. However, while the $m=0$ surface impedance is proportional to a factor of $\ln \frac{d}{b}$ the $m=1$ one is proportional to a smaller factor of $\frac{d^{2}-b^{2}}{d^{2}+b^{2}}$. (b) Longitudinal impedance $(m=0)$ for round chamber (black) and for flat chamber (blue). (c) Transverse impedance for round chamber (black) and horizontal (vertical) impedance multiplied by $\frac{24}{\pi^{2}}$ (green) $\left[\frac{12}{\pi^{2}}\right.$ (blue)] for flat chamber. Note the significant differences for frequencies smaller than $400 \mathrm{MHz}$.
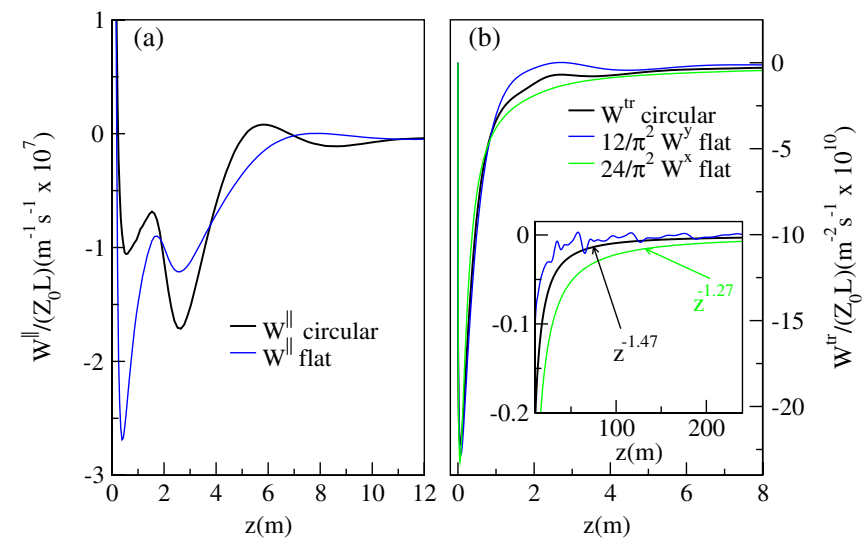

FIG. 7. Comparison between circular (black) laminated chamber and parallel-plane (blue) laminated chamber wake functions. (a) Longitudinal wake. (b) Transverse wake. The horizontal (green) [vertical (blue)] flat chambers wake is multiplied by a factor of $\frac{24}{\pi^{2}}\left(\frac{12}{\pi^{2}}\right)$. Inset: The long distance behavior of transverse wakes. In our example the wake for round chamber decays as $z^{-1.47}$ while the horizontal wake for flat chamber decays as $z^{-1.27}$.

wake. However, the asymptotic behavior at large distances is different, the circular wake going more rapidly to zero than the horizontal wake of the flat chamber. Since the horizontal wake plays a large role in the coherent tune shift in flat chambers [3], as we will discuss in Sec. IV, an approximation which uses the circular wake weighted by the geometric factor $\frac{\pi^{2}}{24}$ to describe the tune behavior in flat chambers would yield erroneous results.

\section{APPLICATION TO THE FERMILAB BOOSTER}

About $60 \%$ of the Fermilab Booster synchrotron ring is made up of laminated iron magnets. There is no pipe in this region, the beam being directly exposed to the laminations. There are two types of combined function gradient magnets, focusing (F) and defocusing (D), with a geometry which in the first approximation can be considered parallel plane [10]. The parameters of the F-magnets are given in the caption of Fig. 4 while the D-magnets have a slightly larger aperture $b=2.9 \mathrm{~cm}$. All the other parameters for the D-magnets are the same as those of the F-magnets.

The longitudinal impedance of the Booster laminated magnets was measured by Crisp and Fellenz [1] using a wire stretched between magnet ends. Compared with previous calculations [5,7], our results show better agreement with the measurement at frequencies below $200 \mathrm{MHz}$. Figure 8 shows the calculated and the measured longitudinal impedance for the F-magnet. In our calculations the real part of the longitudinal impedance shows a broad feature in the interval $100-200 \mathrm{MHz}$ with a maximum at $\sim 280 \Omega$ similar to the measurements, whereas Ng's [7] results display a sharper peak around $50 \mathrm{MHz}$ with a 


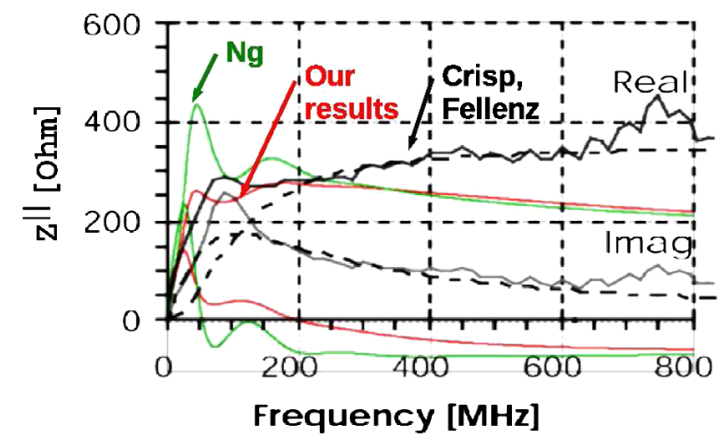

FIG. 8. Longitudinal impedance in the Booster F-magnet. The black and gray lines show the measurements of Crisp and Fellenz [1] (real and, respectively, imaginary impedance). The black dashed lines represent a fitting model of a resistor in parallel with an impedance. The green line are the calculations of $\mathrm{Ng}$ [7]. Our calculations are shown with red lines.

maximum at $\sim 420 \Omega$. Neither our nor the previous calculations show a good agreement of the imaginary part of the impedance with the measurement. However, in our case the impedance is inductive (has a positive imaginary part) up to $200 \mathrm{MHz}$, while in the previous calculations it is inductive only up to $50 \mathrm{MHz}$. At large frequencies the calculations find the imaginary part of the impedance to be small and negative, while the measurements indicate it to be small and positive. While a detailed study of the discrepancies between our calculation and the measurement is beyond the scope of this paper, we note that there are potential shortcomings in both. For example, we assume a parallel-plane geometry for the gradient magnets and a non-frequency-dependent magnetic permeability in the laminations. On the experimental side, the central wire method for impedance measurement has been criticized by several authors who argue that the presence of the wire might disturb the electromagnetic field inside the chamber [7,19].

A measurable effect caused by the large impedance in Booster is the coherent tune shift which increases with the beam intensity. To calculate it we use the wake functions derived in Sec. III D 3 in a complex beam dynamics simulation which employs the SYNERGIA code.

\section{A. SYNERGIA code and beam simulation}

SYNERGIA is an extensible beam dynamics framework utilizing state-of-the-art numerical libraries, solvers, and physics models. SYNERGIA features 3D space charge solvers, impedance modules, and arbitrary-order Lie maps for magnetic optics. A detailed description of SYNERGIA can be found elsewhere [12,13]. In order to model the Booster, we developed new 3D space charge solvers suitable for parallel-plane and rectangular vacuum chambers for SYNERGIA. We also developed new modules to simulate wakefield effects based on the results presented in this paper.
The space charge solvers used in the simulations treat the vacuum chambers as ideal conductors. Thus, the space charge impedance which accounts for the direct interaction between the particles and, as well, for the effect of the image charges and the image currents is included in our simulations. The space-charge impedance effects are important since we present simulations at the Booster injection energy $(\gamma=1.42)$, quite far from the relativistic limit. However, it should mentioned that beside the spacecharge impedance, there are other nonultrarelativistic corrections to the impedance which we neglect here [20].

In SYNERGIA the beam is modeled as a large $\left(\approx 10^{5}-10^{8}\right)$ set of macroparticles. When collective effects are turned off each particle propagates independently in the field created by the machine elements. The single-particle propagation is treated exactly using arbitrary-order maps. Collective effects are incorporated using the split operator method [22]. This implies a large number of momentum kicks during propagation. In brief, the simulation with collective effects is an interleaving sequence of singleparticle propagation followed by a momentum kick, followed by single-particle propagation, followed by a momentum kick, etc. The error is $\mathcal{O}\left(\Delta s^{3}\right)$, where $\Delta s$ is the distance between kicks. The number of kicks is taken to be large enough to reach convergence.

The wake kicks are implemented as follows. Each macroparticle " $i$ " suffers a kick of its momentum proportional to

$$
\begin{gathered}
\Delta p_{z i}=\frac{-q^{2}}{c} \sum_{j>i} W^{\|}\left(z_{j i}\right) \\
\Delta p_{x i}=\frac{-q^{2}}{c} \sum_{j>i}\left[W_{x}^{\perp}\left(z_{j i}\right) X_{j}-W_{x}^{\perp}\left(z_{j i}\right) x_{i}\right] \\
\Delta p_{y i}=\frac{-q^{2}}{c} \sum_{j>i}\left[W_{y}^{\perp}\left(z_{j i}\right) Y_{j}+W_{x}^{\perp}\left(z_{j i}\right) y_{i}\right],
\end{gathered}
$$

where the summation is over all the other particles " $j$ " in front of the particle " $i$." The summation is not restricted only to the bunch " $i$ " belongs to. The wakefield created by all the other bunches as well as the effect of the previous turns are included in this summation.

The space charge kicks are proportional to the electric field created by the other particles in the bunch. The field is calculated using particle in cell Poisson solvers.

The Booster has 24 cells, each including F-magnets and D-magnets and circular pipe drift sections $[2,10]$. We use different wake functions and space charge solvers for the F- and D-magnets. Circular space charge solvers are used for the drift sections.

The simulation is initialized with a six-dimensional Gaussian beam matched for propagation without collective effects. We run 84-bunch (full machine) simulations. The input parameters are $x_{\mathrm{rms}}=0.0086 \mathrm{~m}, y_{\mathrm{rms}}=0.0032 \mathrm{~m}$, and $z_{\mathrm{rms}}=0.88 \mathrm{~m}$. Different beam intensities of up to 
$6 \times 10^{10}$ particles per bunch are considered. The beam energy is $40 \mathrm{MeV}(\gamma=1.42)$. These values are similar to those in the Booster during the experimental runs [10]. Since the experimental data was taken at the injection energy, in the simulations the phasing of the rf cavities is set up so that there is no net acceleration of the beam. To determine the coherent tunes, we measure the position of the beam center at different locations over 1000 turns. The tunes are extracted from the Fourier transform of the beam center displacement as a function of position.

\section{B. Coherent tune shift and comparison with experiment}

A simple inspection of the terms proportional to the trailing particle displacement in Eqs. (2) and (3) (called quadrupole [3] or detuning [16] terms in the literature) reveal that they have different signs for the vertical and the horizontal directions. Keeping in mind that the wake function $W_{x}^{\perp}(z)$ is predominantly negative (see Fig. 5) it can be immediately deduced [3] that the effect of these terms will be to decrease the coherent vertical tune and increase the horizontal one. If the only collective effect acting on the beam were produced by these terms, then one would expect the negative vertical tune shift to be equal to the positive horizontal tune shift. However, the terms proportional to the leading particle displacement as well as the space charge impedance effects act on decreasing both the vertical and the horizontal tunes. Thus, they enhance the decrease of the vertical tune and compete with the horizontal tune enhancement produced by the quadrupole terms. This reasoning is corroborated by recent measurements [2] of the Booster coherent tunes which show a large decrease of the vertical tune and a small positive increase of the horizontal tune with increasing the beam intensity.

The data from the intensity-dependent tune scan shown in Fig. 9 were taken after the transverse Booster tunes had been separated to values of $\nu_{x}=6.7$ (horizontal tune), and $\nu_{y}=6.9$ (vertical tune). This was done by systematically changing the current settings of the quadrupole correction magnets using a method developed and described elsewhere [23]. The tunes were separated to better isolate tune shifts due to collective effects from the influence of machine-dependent mode mixing. Once the tunes were cleanly separated, the beam intensity was varied via injection, while all other machine parameters remained fixed. The tunes were obtained from turn-by-turn Fourier transforms of the 100 horizontal and vertical beam position monitors in the Booster, using an application developed to obtain beam frequency signatures with good signal to noise ratios [24]. In addition, a fast kicker was set to a periodic trigger to ensure the betatron oscillations were large enough to allow a clear tune signature. The horizontal and vertical tunes were examined at 2,3 , and $5.5 \mathrm{~ms}$, during the injection portion of the $33 \mathrm{~ms}$ Booster cycle. The injected beam intensity was varied from $0.9 \times 10^{12}$ to $6.8 \times 10^{12}$ protons.

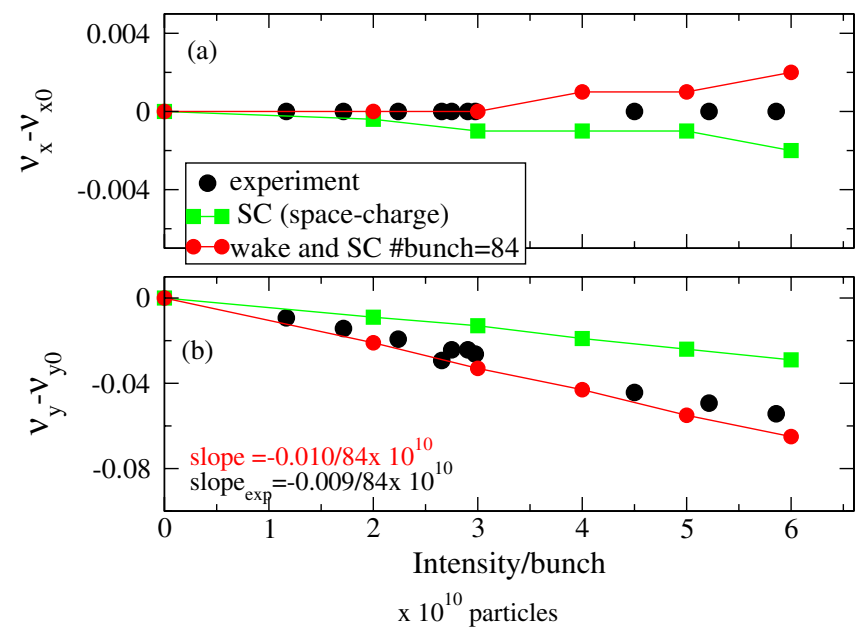

FIG. 9. Coherent horizontal (a) and vertical (b) tune shift versus beam intensity. The estimated tune uncertainty in calculations is less than 0.001 . The vertical tune is decreased while the horizontal tune changes very little. Space charge impedance (SC) decreases both the vertical and horizontal tunes, while the wake decreases the vertical tune and increases the horizontal tune. The calculation are in good agreement with the experiment, the deviation being within $3 \sigma_{\text {meas }}$.

A prior study was done where the tunes were not specifically decoupled, rather, they were highly coupled. It was uncertain how the machine coupling may have affected the evolution of the tunes, so the second study was done with the tunes separated. It was found that coupling effects in the first study were not significant, as the intensity-dependent tune shifts measured in both studies were quite similar [2]. The base machine tunes may be analogously controlled in the beam simulations. Simulations with different base tunes were performed and the results were in agreement with experimental measurements, the horizontal-vertical coupling did not play a significant role in the tune shift analysis.

In Figs. 9(a) and 9(b), simulation of the coherent betatron tune shifts in the horizontal and vertical planes are compared with the experimental data [2]. Notice that the scale for the vertical tune shift is about 20 times larger than the scale for the horizontal tune shift. The red circles are the results of the simulations which include both space charge impedance and the ultrarelativistic wakefields. The agreement with the experimental data is good. Estimating the measurement standard deviation $\sigma_{\text {meas }}$, we find our calculation to be within $3 \sigma_{\text {meas }}$ range. The vertical tunes are decreasing with intensity. As in the experiment, we find that the horizontal tunes do not change significantly with beam intensity, the slope being very small and positive. We also ran simulations where only the space charge impedance was considered. The results of these simulations are shown with green squares. From these results we can conclude that for the vertical case, both the wake and the space charge force decrease the tune, the wake having a 
larger effect. For the horizontal case, the space charge force decreases the tune, while the wake increases it. However, both effects are small.

\section{CONCLUSIONS}

We calculate the impedance and the wake functions for laminated structures with parallel-plane and circular geometries. First, the coupling impedance is derived as a function of the chamber surface impedance. Next, the surface impedance is calculated by solving the Maxwell equations inside the lamination and the crack regions. The impedance is evaluated on a large frequency range, the knowledge of both the low the high frequency behavior being necessary for the calculation of the wake functions.

We present a critical analysis of the resistive-wall impedance for metallic chambers and the approximations involved in its derivations. We show that the commonly used approximations, valid due to the small surface impedance associated with metallic pipes, do not hold for chambers with large surface impedance such as laminated structures. The truncation of the higher-order terms in the surface impedance cannot be done when calculating the impedance. While for the metallic chambers the dependence of the surface impedance on the horizontal wave number (in case of parallel-plane geometry) or on the angular number (in case of circular geometry) is not important, it is essential for the laminated chambers.

As an application, we calculate the coupling impedance and the wake functions for the Fermilab Booster magnets. We used the wake functions in realistic beam simulation using the SYNERGIA code. New modules and subroutines were added to SYNERGIA to take into account the wake interaction. We measured the beam coherent tune shift at injection energy and compare it with experiment [2]. We find that the coherent vertical tune decreases with increasing beam intensity while the horizontal tune is almost constant, in good agreement with the measurements.

\section{ACKNOWLEDGMENTS}

We thank Alexey Burov, Valeri Lebedev, and K. Y. Ng for fruitful discussions. We also thank Yuri Alexahin, William Pellico, and William Marsh for help with beam studies. This work was supported by the United States Department of Energy under Contract No. DE-AC0207CH11359, the ComPASS project, funded through the Scientific Discovery through Advanced Computing program in the DOE Office of High Energy Physics, and NSF Grant No. 0237162. This research used resources of the National Energy Research Scientific Computing Center, which is supported by the Office of Science of the U.S. Department of Energy under Contract No. DE-
AC02-05CH11231, as well as resources of the Argonne Leadership Computing Facility at Argonne National Laboratory, which is supported by the Office of Science of the U.S. Department of Energy under Contract No. DEAC02-06CH11357.

[1] James L. Crisp and Brian Fellenz, Fermilab Report No. TM-2145, 2001.

[2] Daniel McCarron, Ph.D. thesis, Fermilab, 2010.

[3] A. Chao, S. Heifets, and Bruno Zotter, Phys. Rev. ST Accel. Beams 5, 111001 (2002).

[4] S. C. Snowdon, Fermilab Report No. TM-277, 1970.

[5] A. G. Ruggiero, Fermilab Report No. FN-220, 1971.

[6] R. Gluckstern, Fermilab Report No. TM-1374, 1985.

[7] K. Y. Ng, Fermilab Report No. FN-0744, 2004.

[8] A. Chao, Physics of Collective Beam Instabilities in High Energy Accelerators (Wiley, New York, 1993).

[9] A. M. Al-Khateeb, R. W. Hasse, O. Boine-Frankenheim, W. M. Daqa, and I. Hofmann, Phys. Rev. ST Accel. Beams 10, 064401 (2007).

[10] Booster Rookie Book, Fermilab, http://www-bdnew.fnal .gov/operations/rookie_books/rbooks.html.

[11] A. Burov and V. Lebedev, Report No. FERMILAB-TM2492-AD, 2011.

[12] J. Amundson, P. Spentzouris, J. Qiang, and R. Ryne, J. Comput. Phys. 211, 229 (2006).

[13] James Amundson et al., "Synergia2: A Parallel Framework for Beam Dynamics" (to be published).

[14] S. Heifets, A. Wagner, and B. Zotter, Report No. SLAC/ AP110, 1998.

[15] The quadrupole nomenclature used here for the flat chamber wake term proportional to the trailing particle displacement should not be confused with the quadrupole term corresponding to the angular number $m=2$ in chambers with circular geometry. The latter is second order in the beam displacement.

[16] A. Burov and V. Lebedev, in Proceedings of the 8th European Particle Accelerator Conference, Paris, 2002 (EPS-IGA and CERN, Geneva, 2002), p. 1445.

[17] S. A. Heifets and S. A. Kheifets, Rev. Mod. Phys. 63, 631 (1991).

[18] J.D. Jackson, Classical Electrodynamics (Wiley, New York, 1999), 3rd ed.

[19] A. Chao, Report No. SLAC-PUB 9574, 2002.

[20] For example, see the nonultrarelativistic calculation of the resistive-wall impedance in circular pipes in Ref. [21].

[21] F. Zimmermann and K. Oide, Phys. Rev. ST Accel. Beams 7, 044201 (2004).

[22] H. Yoshida, Phys. Lett. A 150, 262 (1990).

[23] Y. Alexahin and E. Gianfelice-Wendt, Report No. Fermilab-pub-06-093-AD, 2006.

[24] Y. Alexahin, E. Gianfelice-Wendt, and W. Marsh, Reports No. IPAC-2010-MOPE084 and No. Fermilab-conf-10135-APC, 2010. 